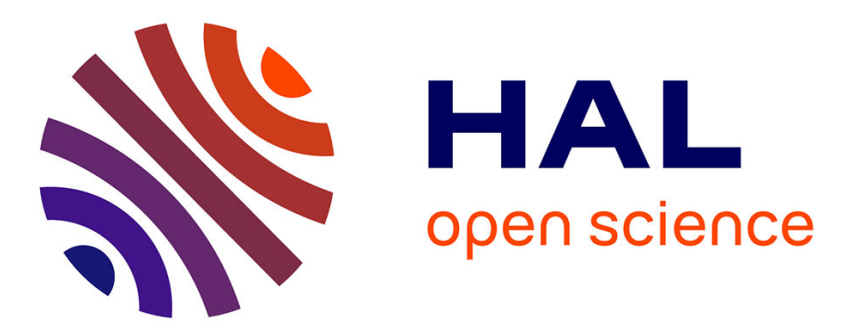

\title{
Evolutionary costs and benefits of infection with diverse strains of Spiroplasma in pea aphids
}

Hugo Mathe-Hubert, Heidi Kaech, Pravin Ganesanandamoorthy, Christoph Vorburger

\section{- To cite this version:}

Hugo Mathe-Hubert, Heidi Kaech, Pravin Ganesanandamoorthy, Christoph Vorburger. Evolutionary costs and benefits of infection with diverse strains of Spiroplasma in pea aphids. Evolution International Journal of Organic Evolution, 2019, 10.1111/evo.13740 . hal-02168387

\section{HAL Id: hal-02168387 \\ https://hal.science/hal-02168387}

Submitted on 28 Jun 2019

HAL is a multi-disciplinary open access archive for the deposit and dissemination of scientific research documents, whether they are published or not. The documents may come from teaching and research institutions in France or abroad, or from public or private research centers.
L'archive ouverte pluridisciplinaire HAL, est destinée au dépôt et à la diffusion de documents scientifiques de niveau recherche, publiés ou non, émanant des établissements d'enseignement et de recherche français ou étrangers, des laboratoires publics ou privés. 


\title{
Evolutionary costs and benefits of infection with diverse strains of Spiroplasma in pea aphids
}

\author{
Hugo Math 'e-Hubert, ${ }^{1,2,3}$ Heidi Kaech, ${ }^{1,4}$ Pravin Ganesanandamoorthy, ${ }^{1}$ and Christoph Vorburger ${ }^{1,4}$ \\ ${ }^{1}$ Eawag, Swiss Federal Institute of Aquatic Science and Technology, "Uberlandstrasse 1338600 D "ubendorf, \\ Switzerland '2 Current Address: LIEC UMR 7360, Universite' de Lorraine and CNRS, Metz, France \\ ${ }^{3}$ E-mail: hugomh@gmx.fr \\ ${ }^{4}$ Institute of Integrative Biology, Department of Environmental Systems Science, ETH Zürich, Universitätsstrasse 168092 \\ Zürich, Switzerland
}

Received October 31, 2018

Accepted March 28, 2019

\begin{abstract}
The heritable endosymbiont Spiroplasma infects many insects and has repeatedly evolved the ability to protect its hosts against different parasites. Defenses do not come for free to the host, and theory predicts that more costly symbionts need to provide stronger benefits to persist in host populations. We investigated the costs and benefits of Spiroplasma infections in pea aphids (Acyrthosiphon pisum), testing 12 bacterial strains from three different clades. Virtually all strains decreased aphid lifespan and reproduction, but only two had a (weak) protective effect against the parasitoid Aphidius ervi, an important natural enemy of pea aphids. Spiroplasma-induced fitness costs were variable, with strains from the most slowly evolving clade reaching higher titers and curtailing aphid lifespan more strongly than other strains. Some Spiroplasma strains shared their host with a second endosymbiont, Regiella insecticola. Although the result of an unfortunate handling error, these co-infections proved instructive, because they showed that the cost of infection with Spiroplasma may be attenuated in the presence of Regiella. These results suggest that mechanisms other than protection against $A$. ervi maintain pea aphid infections with diverse strains of Spiroplasma, and that studying them in isolation will not provide a complete picture of their effects on host fitness.
\end{abstract}

KEY WORDS: Acyrthosiphon pisum, cost of infection, defensive symbiosis, facultative secondary symbionts, lifespan, parasitoid.

\section{Introduction}

Microbial endosymbionts of eukaryotes are ubiquitous, and have often become heritable through the evolution of mother-tooffspring transmission. Large-scale screens for symbionts like Wolbachia or Cardinium suggest that the majority of arthropod species are likely to carry heritable infections with endosymbionts (Zchori-Fein and Perlman 2004; Hilgenboecker et al. 2008). Microbial symbionts may provide their hosts with essential nutrients, especially in species with very imbalanced diets such as blood feeders like the tsetse fly (Chen et al. 1999) or phloem feeders like aphids (Douglas 1998). Some of these symbioses are ancient and have evolved to the point that the host is unable to survive without its bacterial partner, which is referred to as an obligate symbiont (Wernegreen 2002; Moran et al. 2008). Other endosymbionts are facultative associates for the host and not strictly required for host survival. These are referred to as secondary symbionts. A single arthropod species can host multiple species of secondary symbionts, but each symbiont typically infects only a part of the host population (e.g., Chiel et al. 2007; Ferrari et al. 2012). Additional variation may be present within symbionts. A secondary symbiont species infecting a particular host species often comprises multiple distinguishable strains (Raychoudhury et al. 2009; Ferrari et al. 2012; Russell et al. 2013). Explaining the evolutionary persistence and the high diversity of secondary symbionts in host populations requires an understanding of how different symbionts counterbalance the costs they impose on their host (Heath and Stinchcombe 2014).

One way for maternally transmitted symbionts to spread in a host population is to manipulate the host's reproduction in a way that favors symbiont transmission. Reproductive manipulation has 
evolved repeatedly in endosymbiotic bacteria like Wolbachia, Arsenophonus, Cardinium, Rickettsia, or Spiroplasma (Duron et al. 2008). It can act via the induction of cytoplasmic incompatibility, male-killing, parthenogenesis, or the feminization of genetically male offspring (Werren et al. 2008).

In addition to reproductive manipulation, heritable symbionts can spread if they provide their host with an evolutionary benefit. This strategy is not mutually exclusive with reproductive manipulation. An important class of evolutionary benefits that has evolved repeatedly is protection against natural enemies, that is, defensive symbiosis (Oliver and Moran 2009; McLean 2019). Multiple species of secondary symbionts increase the resistance of aphids against parasitoid wasps and pathogenic fungi (Oliver et al. 2003; Scarborough et al. 2005; Vorburger et al. 2010; Łukasik et al. 2013), certain strains of Spiroplasma can protect flies against parasitoid wasps or parasitic nematodes (Jaenike et al. 2010; Xie et al. 2010; Paredes et al. 2016), and Wolbachia can reduce viral infection in flies and other insects (Hedges et al. 2008; Teixeira et al. 2008; Bian et al. 2010). So why do these seemingly beneficial symbionts not go to fixation in host populations?

Most general explanations assume trade-offs between the benefits provided by the symbiont and the costs associated with its possession, acting in combination with environmental heterogeneity. For example, the secondary symbiont Hamiltonella defensa (Moran and Russell 2005) can protect different aphid species against parasitism (Oliver et al. 2003; Schmid et al. 2012; Asplen et al. 2014), but $H$. defensa is selected against in the absence of parasitoids (Oliver et al. 2008), possibly because of the reductions in host lifespan and lifetime reproduction or in nymphal growth it induces (Vorburger and Gouskov 2011; Leybourne et al. 2018). Temporal and spatial variation in the risk of parasitism may thus maintain coexistence between infected and uninfected hosts. Similarly, species and strain diversity may partly be explained by unequal effects against different natural enemies. For H. defensa, several studies have shown that protection of aphids against parasitoid wasps can be highly specific (reviewed in Vorburger 2014). A given strain of $H$. defensa can provide effective protection against some parasitoid species but not against others (Asplen et al. 2014; Cayetano and Vorburger 2014; McLean and Godfray, 2015, 2017; Martinez et al. 2016), and this specificity can even extend to interactions within species. In black bean aphids (Aphis fabae), particular isolates of $H$. defensa protect strongly against some parasitoid genotypes but not or only weakly against other parasitoid genotypes, leading to strong genotypeby-genotype interactions between parasitoids and the hosts' defensive symbionts (Schmid et al. 2012; Cayetano and Vorburger 2013; Vorburger and Rouchet 2016). Similar genotype-specificity is observed in the interaction between the fungal pathogen Pandora neoaphidis and the secondary symbiont Regiella insecticola, which protects pea aphids (Acyrthosiphon pisum) against fungal infection (Parker et al. 2017). Variation in the local parasitoid and pathogen community may thus select for different secondary symbionts, and genotype-by-genotype specificity may further maintain strain variation via negative frequency-dependent selection (Kwiatkowski et al. 2012; Heath and Stinchcombe 2014).

A promising system to investigate the evolutionary maintenance of symbiont strain diversity are bacteria of the genus Spiroplasma. These helical, cell wall-less bacteria belong to the class Mollicutes within the phylum Firmicutes (Gasparich et al. 2004). Spiroplasma bacteria are generally associated with arthropods, but they differ widely in their modes of transmission and their phenotypic effects on the hosts. Some are virulent, horizontally transmitted pathogens of insects and crustaceans that cause problems in apiculture and aquaculture (Clark et al. 1985; Wang et al. 2005), some are damaging plant pathogens that are vectored by phloem-feeding insects (Bové et al. 2003), and many are vertically transmitted endosymbionts (Williamson et al. 1998; Watts et al. 2009). It is estimated that between 5\% and $10 \%$ of insects carry heritable infections with Spiroplasma (Duron et al. 2008). Similar to other heritable endosymbionts, some Spiroplasma have evolved the ability to defend their hosts against other infections (Ballinger and Perlman 2018). For example, the male killing strain MSRO of $S$. poulsonii protects Drosophila melanogaster against parasitoid wasps (Xie et al. 2014; Paredes et al. 2016), illustrating that reproductive manipulation and protection are not mutually exclusive strategies of symbionts to spread in host populations. In the fungus-feeding D. neotestacea, infection with Spiroplasma induces tolerance to the parasitic nematode Howardula aoronymphium (Jaenike et al. 2010). In pea aphids, Spiroplasma has been shown to protect against fungal infections (Łukasik et al. 2013a), and there is evidence for male-killing by at least one strain (Simon et al. 2011).

Spiroplasma infecting European pea aphids are subdivided into at least three clades that are similarly abundant in aphids feeding on different host plants, but share their hosts with different symbiont communities and have a different rate of molecular evolution, suggesting their maintenance in pea aphids might rely on different eco-evolutionary strategies (Mathé-Hubert et al. 2018). Here, we provide insights in the ecology and evolution of these three clades. We investigate if protection against the pea aphid's main parasitoid Aphidius ervi might contribute to the evolutionary persistence of Spiroplasma in this species. Twelve Spiroplasma strains, evenly spread across the three clades, were tested for their ability to protect against three different lines of the parasitoid $A$. ervi, and we estimated Spiroplasma density in 10- and 20-dayold aphids, as well as Spiroplasma's effects on aphid fitness in the absence of parasitoids. Although two of the 12 Spiroplasma strains reduced aphid parasitism by at least one of three parasitoid lines, there was no global effect of Spiroplasma on the parasitism 


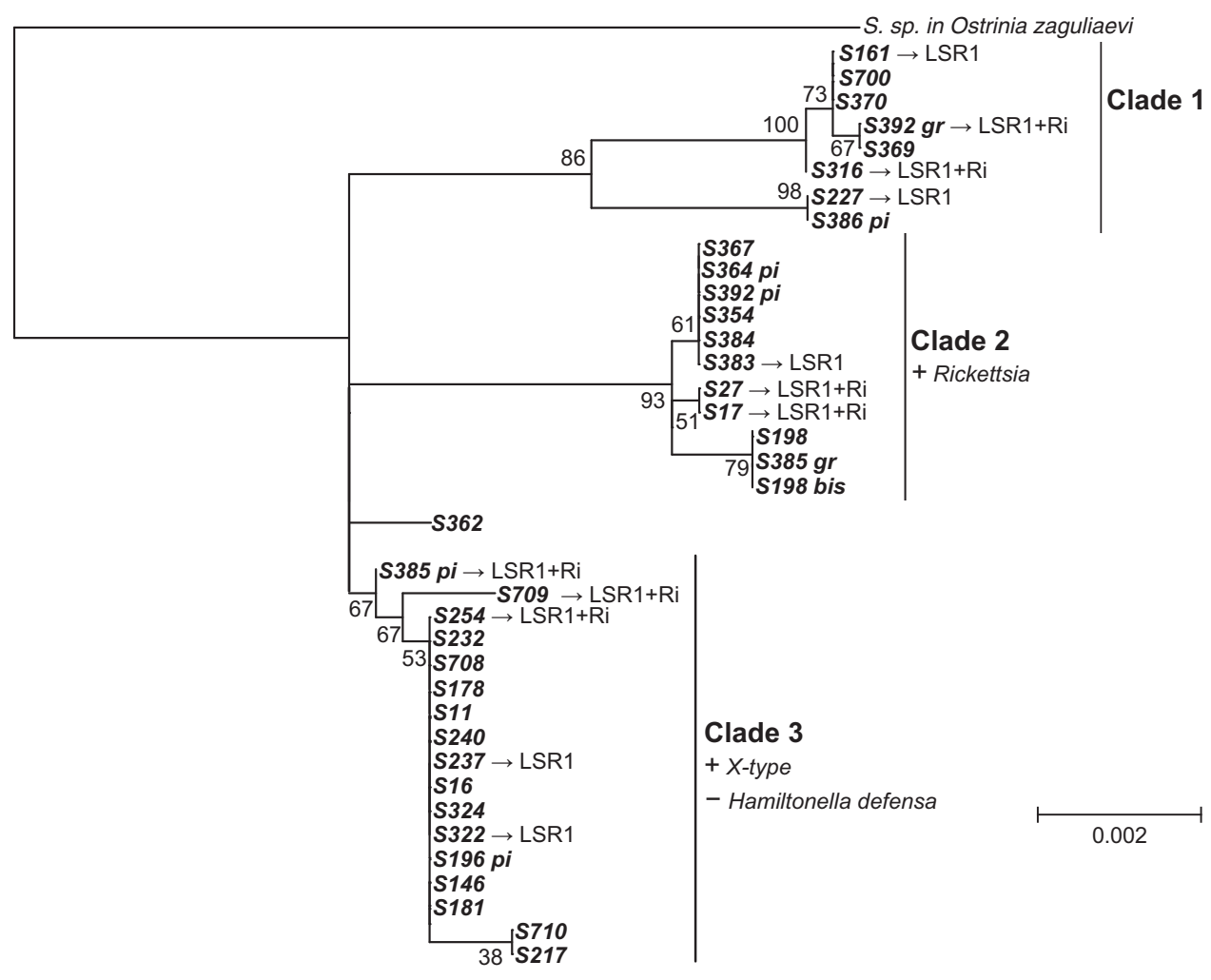

Figure 1. Spiroplasma phylogeny. Phylogeny modified from Mathé-Hubert et al. (2018). Strains selected for the phenotyping experiments are followed by an arrow indicating whether they were transfected into pea aphid clone LSR1 containing R. insecticola ( $\rightarrow$ LSR1+Ri) or not $(\rightarrow$ LSR1). The clade to which the Spiroplasma strain belongs and the other symbionts with which strains of that clade are typically associated $(+)$ or not associated $(-)$ is indicated on the right. Values close to the nodes are bootstrap values. The scale bar indicates the substitution rates.

success. All Spiroplasma strains curtailed aphid lifespan and lifetime reproduction to various extents and the benefit provided by the two protective Spiroplasma strains is unlikely to counterbalance their cost. This suggests that Spiroplasma infection in pea aphids is maintained by another mechanism than the protection against A. ervi. An analysis of phylogenetic signal in the phenotypic data further revealed that the most slowly evolving of the three Spiroplasma clades attains the highest titer in aphids and reduces aphid lifespan more strongly than the other clades.

\section{Material and Methods INSECT LINES}

To investigate phenotypic effects of Spiroplasma infections, we used the European field survey and the phylogeny of Spiroplasma from pea aphids reported in Mathé-Hubert et al. (2018) to select 12 strains that are well spread across the phylogeny (Fig. 1). To control for the effect of aphid genotype, the selected Spiroplasma strains were transfected from their original host clones (the donors) into a common recipient clone called LSR1. This clone was originally collected in a field of alfalfa (Medicago sativa) near Ithaca, New York, in 1998 (Caillaud et al. 2002), and its genome has been sequenced for the pea aphid genome project (The International Aphid Genomics Consortium 2010). Four Spiroplasma strains were transfected into LSR1 at the University of Oxford, U.K., and kindly provided to us by Ailsa McLean. The remaining transfections were carried out in our laboratory at Eawag, Switzerland. Before transfection of Spiroplasma with a microinjection pump (FemtoJet, Eppendorf) as described by Vorburger et al. (2010), the donor aphids were cured from all other secondary symbionts by feeding them on a mixture of antibiotics as described in McLean et al. (2011). For strain S383, this protocol failed to remove a co-infection with Hamiltonella defensa in the donor clone. We thus merged the curing and transfection step by injecting recipients with a small amount of a $20 \mathrm{mg} / \mathrm{mL}$ solution of the antibiotic cefotaxime, using a needle that was immersed into the donor's hemolymph prior to injection. This procedure succeeded in transmitting just Spiroplasma to the recipient clone. Depending on the strains, the transfections happened between 10 and $\sim 150$ generations before the experiments.

Although prior to transfections we had reconfirmed the genotypes and the secondary symbiont infections of the donors and the 
recipient clone with microsatellites and diagnostic PCRs, respectively, a handling error must have occurred between these checks and the actual transfections, such that we used a $R$. insecticolainfected sub-line of clone LSR1 (LSR+Ri) as recipient rather than the sub-line without any secondary symbionts. As a consequence, seven of the 12 newly transfected sub-lines carried a co-infection with $R$. insecticola in addition to the different Spiroplasma strains. Only sub-line LSR1+S383 (presumably due to the simultaneous injection of an antibiotic-see above), the four sub-lines provided by the University of Oxford, and the secondary endosymbiontfree control did not carry $R$. insecticola. Figure 1 summarizes the infection status of each sub-line. That the $R$. insecticola-infected sublines indeed belonged to clone LSR1 was confirmed by microsatellite genotyping, and sequencing of five bacterial genes (accD, gyrB, murE, recJ, and rpoS; Henry et al. 2013) identified the co-infecting $R$. insecticola as a strain previously shown to provide no protection against $A$. ervi in pea aphids (Oliver et al. 2003; Hansen et al. 2012). Because we discovered this error only after all phenotyping experiments had been completed, we had to account statistically for the presence of $R$. insecticola during data analysis (see below).

\section{ACCOUNTING STATISTICALLY FOR CO-INFECTIONS WITH R. insecticola}

For the three experiments described hereafter, we handled the presence of $R$. insecticola according to the following logic: We estimated the average effect of $R$. insecticola on each trait we analyzed and then used this estimate as an offset to correct for its presence in the coinfected sublines. Specifically, we first fit a "Regiella" model devised to estimate the average effect of $R$. insecticola in the presence of a Spiroplasma strain. In addition to the variables specific to each experiment (described in the corresponding sections), this model contains two dummy variables as fixed effects describing the presence (1) or absence (0) of Spiroplasma and $R$. insecticola (variables $S_{i}$ and $R_{i}$, respectively) and a random interaction between the aphid subline $(S U B)$ and the fixed effect $S$. This random effect follows a normal distribution of mean zero and standard deviation $\sigma$. Mathematically, this gives

$$
\begin{aligned}
Y_{i}= & \text { Int }+\alpha \times R_{i}+\beta \times S_{i}+S_{i} \times S U B \\
& +e_{i} ; S U B \sim N(0, \sigma)
\end{aligned}
$$

where $Y_{i}$ is the transformed explained variable, $\mathrm{e}_{i}$ are the residuals estimated by the models together with the coefficients of the fixed effects ( $\alpha$ and $\beta$ ) and the standard deviation $(\sigma)$. Because we used dummy variables, the intercept of the model (Int) is the mean of the control sub-line containing neither Spiroplasma nor $R$. insecticola. The coefficients $\alpha$ and $\beta$ are the estimated mean effects of $R$. insecticola and Spiroplasma, and the random interaction between the sub-line and $S$ accounts for the heterogeneity induced by the different Spiroplasma strains. This estimation of the effect of $R$. insecticola assumes that on average the Spiroplasma strains that are alone have the same effect as the Spiroplama strains that are with $R$. insecticola. The estimated effects of $R$. insecticola (coefficient $\alpha$ in eq. 1) is then used to construct an offset (Hutchinson and Holtman 2005) for the second "Spiroplasma" model estimating the effect of each Spiroplasma strain. This offset takes the value $\alpha$ when $R$. insecticola is present and 0 when it is absent. The "Spiroplasma" model contains the aphid sub-line as a fixed effect. Thus, for the sub-lines not containing $R$. insecticola, there is no offset and each coefficient describes the effect of the sub-line's Spiroplasma strain, and for the sub-lines containing $R$. insecticola, the estimated effect of $R$. insecticola in the presence of Spiroplasma is absorbed by the offset, and each coefficient describes the effect of the corresponding Spiroplasma strain plus its eventual interaction with $R$. insecticola.

\section{EXPERIMENT 1: EFFECT OF SPIROPLASMA ON A. ervi PARASITISM}

We investigated the effect of the 12 Spiroplasma strains on the parasitism success of three different lines of the parasitoid wasp A. ervi (lines "B," "D," and "K"). We established the line "D" using wasps sampled in July 2015 at two sites in southern Germany during the field survey reported in Mathé-Hubert et al. (2018). This wasp line has been maintained in the laboratory for approximately 40 generations prior to the experiment. The two other A. ervi lines " $\mathrm{K}$ " and "B" were commercially supplied by the biocontrol companies Koppert (Berkel en Rodenrijs, the Netherlands) and Biobest (Westerlo, Belgium), and were reared in the laboratory for one and two generations before the experiment, respectively. We used three different lines of parasitoids to increase our chances of detecting any protective effects of Spiroplasma, since previous studies on another bacterial endosymbiont, $H$. defensa, have shown that the protection afforded by the symbiont can depend on the parasitoid's genotype (e.g., Schmid et al. 2012; Cayetano and Vorburger 2013). All wasps were bred on the same pea aphid clone (lab ID A06-01) that was free of protective endosymbionts and different from the clone used in experiments (LSR1).

Parasitism success was measured using a factorial design in which the 13 aphid sub-lines (12 Spiroplasma-infected sub-lines plus uninfected control) were exposed to all three parasitoid lines in six randomized complete blocks. To prevent maternal effects carried over from the aphid stock cultures influencing our results, each of the 234 replicates (13 aphid sub-lines $\times 3$ wasp lines $\times 6$ replicates) was reared independently on seedlings of broad bean (Vicia faba) for one generation before individuals of the second generation were tested. To start the test generation, five adults from each replicate were used to obtain age-synchronized offspring born within $24 \mathrm{~h}$. At the age of 2-3 days, 20 nymphs 
per replicate were placed on a new plant and exposed to a single female wasp ( $\sim 2$ days old) for $5 \mathrm{~h}$. Because a few aphid nymphs were harmed during the exposure to wasps, the number of nymphs alive one day after the exposure was recorded. The proportion of these surviving nymphs that were successfully parasitized and transformed into mummies (parasitoid pupae within the dead aphid's exoskeleton) was recorded 11 days after exposure to parasitoids. The proportion of mummies from which adult wasps had emerged successfully (proportion emerged) was recorded 20 days after exposure. We conducted this experiment at $22^{\circ} \mathrm{C}$ under a $16-\mathrm{h}$ photoperiod.

For each of the two variables, proportion mummified and proportion emerged, we fitted the "Regiella" and "Spiroplasma" models as described above. Both models additionally contained the wasp line as a fixed effect as well as its interaction with the dummy variables " $R$ " and " $S$ " for the model "Regiella" and with the aphid sub-line for the model "Spiroplasma." Both models also contained the random variable "Block."

If for the "Spiroplasma" model the wasp line $\times$ aphid sub-line interaction was significant, we re-fitted one model per wasp line to test for overall variation among aphid sub-lines and to assess the effect of each Spiroplasma strain using a Student's $t$-test. These tests compare each Spiroplasma-infected sub-line to the uninfected control sub-line by assessing the significance of the coefficients of the variable "aphid sub-line." We then used the package "multcomp" (Hothorn et al. 2008) to assess for each of the models fitted to one wasp line that Spiroplasma strains had a significant effect after accounting for multiple testing. When the wasp line $x$ aphid sub-line interaction was not significant, we refitted the model without the interaction to test for the effect of each Spiroplasma strain.

Since the explained variables were proportions, we first fitted them using binomial GLMMs ("Ime4" R package; Bates et al. 2014), which were strongly overdispersed. The attempt to mitigate overdispersion with the "observation level random effect" approach (Harrison 2015) resulted in severe underdispersion. Thus, we fitted LMMs to the logit transformed proportions (Warton and Hui 2011). To assess the significance of the main effects, we used the "mixed" function of the "afex" R package (version 0.18 ) to perform an $F$-test with the Kenward-Roger approximation for degrees of freedom (Halekoh and Højsgaard 2014). All statistical analyses were performed using the software $\mathrm{R}$ (version 3.5.2).

\section{EXPERIMENT 2: FITNESS COST OF SPIROPLASMA}

We assessed the fitness cost of Spiroplasma strains by measuring their effects on several life-history traits of their host using the surplus of nymphs produced in experiment 1 : For three of the six blocks, each containing three replicates of every aphid sub-line, we kept all leftover nymphs until they were 6 days old. Then, for each of the 117 replicates (13 aphid sub-lines $\times 9$ replicates organized into 3 blocks), we selected two young aphids for the life table experiment. In $40 \%$ of the cases, one of the two aphids developed wings. They were excluded from the experiment. The 199 wingless aphids were raised individually on broad bean seedlings until their death. Every week, we moved the aphids to a new 9-day-old plant, and recorded the number of offspring they had produced on the former plant. We recorded the survival of the monitored aphids three times a week. The experiment was carried out at $18^{\circ} \mathrm{C}$ and under a 16 -h photoperiod.

We used the life table data to estimate four fitness-related life history traits. The first two are lifetime reproduction (total number of offspring) and lifespan. We also computed the mean reproductive age of each aphid (age of mother at each birth, averaged across all offspring births). In comparison to the lifetime reproduction, the mean reproductive age accounts for the fact that two genotypes with the same lifetime reproduction could have different fitness if one of them produced its offspring earlier than the other. The fourth variable was the intrinsic growth rate, that is, the constant $r$ in the equation describing population growth in an unlimited environment: $N_{t}=N_{0} e^{r t}$. The procedure to calculate it is described in Birch (1948). This variable combines the information of the number of offspring and of the age of the mother when the offspring are produced.

To each of these four fitness-related variables, we fitted the 'Regiella' and 'Spiroplasma' models. Both models also included the random variables block and replicate, the latter accounting for the non-independence of the two individuals taken from the same colony of experiment 1 . The test procedure for these four variables is the same as described for experiment 1, except that a box-cox transformation was used to achieve normality of residuals and homoscedasticity instead of the logit function. For the survival data, we used the "coxme" R package (version 2.2-5) to fit a cox model (Therneau 2015a). For this survival analysis, we checked the assumption of proportional hazard using the "cox.zph" function of the package "survival" (Therneau 2015b; version 2.43-3) and the "survplot" function of the package "rms" (Harrell 2017; version 5.1-2), with the argument "loglog" set to true. As in experiment 1 , this model assessed the overall variation among aphid sub-lines and compared each Spiroplasma-infected sub-line to the uninfected control.

\section{EXPERIMENT 3: VARIATION IN SPIROPLASMA DENSITY}

The density of Spiroplasma within its host may influence both the cost Spiroplasma inflicts on the aphid and the parasitism by A. ervi. Thus, we measured the density of Spiroplasma in 10- and 20-day-old aphids using quantitative PCR (qPCR). For each combination of age and strain, we measured five biological replicates, each consisting of a pool of three aphids that were 
reared on a 9-day-old plant, a different plant being used for each biological replicate. The biological replicates were reared within a single tray on randomized positions. DNA was extracted using either the Qiagen "DNeasy 96 Blood \& Tissue Kit" (extraction in plates; $N=104$ samples) or the Qiagen "DNeasy Blood \& Tissue Kit" (extraction in tubes; $N=16$ samples) after the aphids had been crushed by shaking them 30 times per second for $40 \mathrm{sec}$ with two glass marbles of $2 \mathrm{~mm} \varnothing$ on a bead mill (TissueLyser II, Qiagen). These extractions typically yield approximately $5 \mu \mathrm{g}$ of DNA in $200 \mu \mathrm{L}$.

For each pool of three aphids, the number of Spiroplasma and aphid gene copies were estimated using a Roche LightCycler 480 2.0. Each $12.5 \mu \mathrm{L}$ of qPCR reaction included $6.25 \mu \mathrm{L}$ of GoTaq ${ }^{\circledR}$ qPCR Master Mix, $1.25 \mu 1$ Dnase free Water, $2.5 \mu 1$ of DNA template and $1.25 \mu \mathrm{L}$ each of the $4.5 \mu \mathrm{M}$ forward and reverse primers. Primers for the Spiroplasma dnaA gene were DnaA_F 5'-AAT GCT TGG ATC ATA ATT TAA AGA C- $3^{\prime}$ and DnaA_R 5'-GTT TTG AAG AAA GAA ATG TTT CAA G- $3^{\prime}$. Primers for the $A$. pisum Ef1a gene were Ef1a_F 5'-TAG CAG TTA CAT CAA GAA AAT CGG-3' and Ef1a_R 5'-ATG TTG TCT CCA TTC CAT CCA G-3'. Cycling conditions are described in Table S2. Gene copy numbers were estimated with reference to a standard curve generated with serial dilutions of a synthetic standard. We did not standardize the overall DNA concentrations among samples because we were mainly interested in the Spiroplasma titers (number of Spiroplasma gene copies relative to aphid gene copies), and because the randomization of biological replicates safeguarded us against any unwanted biases. However, to improve the precision of the measurements, samples with a very high concentration were re-run after a dilution devised to yield an expected $\mathrm{Cp}$ around 20. For each sample, the number of gene copies per aphid individual was calculated from the average of triplicate qPCR reactions.

Because the format of the extraction kit (DNeasy 96 Blood \& Tissue Kit [plate format] vs. DNeasy Blood \& Tissue Kit [individual tubes]) had a strong effect on the estimated number of aphid gene copies and a minor effect on the estimated number of Spiroplasma gene copies (Fig. S1), we removed the estimated effect of the extraction kit using the function "removeBatchEffect" of the package "limma" (Smyth 2005, version 3.38.3) prior to further analyses. These corrected numbers of Spiroplasma and aphid gene copies per individual are indicated as \#Spiroplasma dnaA and \#aphid EF1a, respectively. The number of Spiroplasma gene copies per aphid gene copy is defined as \#Spiroplasma dnaA/\#aphid EF1a.

We fitted the "Regiella" and "Spiroplasma" models to each of the three variables \#Spiroplasma dnaA, \#aphid EF1a, and \#Spiroplasma dnaA/\#aphid EF1a. Since the uninfected sub-line was not included in this part of the study, the "Regiella" model did not contain the dummy variable " $S$ " (i.e., all the investigated sub- lines carried Spiroplasma). The "Regiella" and "Spiroplasma" models additionally contained the aphid age (10 or 20 days) as a fixed effect as well as its interaction with the dummy variables " $R$ " for the model "Regiella" and with the aphid sub-line for the model "Spiroplasma." The test procedure is the same a described for experiment 1 , except that since there is no random effect in the "Spiroplasma" model, the main effects were tested using $F$-tests, and we additionally fitted a model separately for each aphid age to assess differences between sub-lines using Tukey's tests.

\section{PHYLOGENETIC ANALYSES}

We performed two analyses using the phylogeny of Spiroplasma strains inferred by Mathé-Hubert et al. (2018). This phylogeny (Fig. 1) showed that Spiroplasma of pea aphids are divided into at least three clades. The first analysis tested if the Spiroplasma induced phenotypes correlate with the phylogeny (phylogenetic inertia), which is expected if these phenotypes evolve slowly in comparison to the sequences used to discriminate Spiroplasma strains. Such phylogenetic inertia would mean that in pea aphids, different clades of Spiroplasma have different effects on their host. Then we tested if clade 3 , which appears to have short branches in the phylogeny, has a lower rate of molecular evolution than the two other clades.

To test for phylogenetic inertia and to investigate the links among the Spiroplasma induced phenotypes, we characterized the variation in the effects of Spiroplasma strains on the phenotype of their host by the coefficients of the "Spiroplasma" models from the three experiments. These coefficients were used rather than the raw data because they represent the estimated effect of Spiroplasma after accounting for Regiella. A PCA was used to summarize this phenotypic variation. In this PCA, individuals (rows) are the Spiroplasma strains that are characterized by the coefficients of the "Spiroplasma" models on the different traits (i.e., one column per trait). These traits (columns) were weighted to ensure that the three experiments had the same weight whatever the number of traits we measured during the experiment. Since the intrinsic growth rate is a composite variable of other variables, it was included in the PCA as a supplementary variable: it was projected onto the PCA after the PCA was inferred. We tested for phylogenetic inertia on the first two PCA axes that jointly explained $57.03 \%$ of the phenotypic variation. Two measures of phylogenetic inertia are generally recommended, the lambda index and Abouheif's $C_{\text {mean }}$ index (Münkemüller et al. 2012). For our phylogeny, the latter has more power (Fig. S2). Hence, we used $\mathrm{C}_{\text {mean }}$ to measure phylogenetic inertia and tested its significance by performing 10000 randomizations using the package "phylosignal" (Keck et al. 2016).

For the Spiroplasma strains that share their host with $R$. insecticola, the coefficients used in the analysis describe the 
Table 1. Analyses of parasitism by the parasitoid wasp Aphidius ervi.

\begin{tabular}{|c|c|c|c|c|c|c|c|}
\hline \multirow{2}{*}{$\begin{array}{l}\text { Explained variable } \\
\text { Proportion mummified }\end{array}$} & \multicolumn{2}{|c|}{ Model } & \multirow{2}{*}{$\begin{array}{l}\text { Wasp lines } \\
\text { All }\end{array}$} & \multirow{2}{*}{$\frac{\text { Effect }}{\text { Wasp }}$} & \multirow{2}{*}{$\frac{\mathrm{df}}{2,210}$} & \multirow{2}{*}{$\begin{array}{l}\mathrm{F} \\
5.98\end{array}$} & \multirow{2}{*}{$\frac{\text { p.value }}{0.003^{* *}}$} \\
\hline & 1 & "Regiella" & & & & & \\
\hline & & & & Spiro. $(0 / 1)$ & $1,78.73$ & 0.09 & 0.763 \\
\hline & & & & Regi. $(0 / 1)$ & 1,10 & 1.83 & 0.206 \\
\hline & & & & Wasp $\times$ Spiro & 2,210 & 0.44 & 0.646 \\
\hline & & & & Wasp $\times$ Regi. & 2,210 & 0.18 & 0.836 \\
\hline & 2 & "Spiroplasma" & All & Wasp & 2,190 & 58.82 & $<0.001^{* * *}$ \\
\hline & & & & Sub-line & 12,190 & 2.17 & $0.009^{* *}$ \\
\hline & & & & Wasp $\times$ Sub-line & 24,190 & 1.57 & 0.050 \\
\hline & 3 & "Spiroplasma" & B & Sub-line & 12,60 & 4.91 & $<0.001^{* * *}$ \\
\hline & 4 & "Spiroplasma" & $\mathrm{D}$ & Sub-line & 12,60 & 0.73 & 0.72 \\
\hline & 5 & "Spiroplasma" & $\mathrm{K}$ & Sub-line & 12,60 & 1.25 & 0.273 \\
\hline \multirow[t]{8}{*}{ Proportion emerged } & 6 & "Regiella" & All & Wasp & $2,185.32$ & 0.64 & 0.528 \\
\hline & & & & Spiro. $(0 / 1)$ & $1,160.60$ & 0.28 & 0.594 \\
\hline & & & & Regi. $(0 / 1)$ & $1,10.53$ & 3.08 & 0.108 \\
\hline & & & & Wasp $\times$ Spiro . & $2,186.07$ & 0.27 & 0.758 \\
\hline & & & & Wasp $\times$ Regi. & $2,186.91$ & 0.26 & 0.770 \\
\hline & 7 & "Spiroplasma" & All & Wasp & $2,164.64$ & 5.63 & $0.004^{* *}$ \\
\hline & & & & Sub-line & $12,164.70$ & 0.55 & 0.880 \\
\hline & & & & Wasp $\times$ Sub-line & $24,164.65$ & 0.87 & 0.638 \\
\hline
\end{tabular}

Models 1-5 explain the proportion of the sets of 20 nymphs exposed to one wasp that were mummified. Models 6 and 7 explain the proportion of mummies from which a wasp emerged. Models 1 and 6 estimate the effect of $\boldsymbol{R}$. insecticola and were used to build the offsets correcting for the presence of $\boldsymbol{R}$. insecticola in the other models. Models 3-5 investigate the interaction between wasp line $x$ aphid sub-line that is significant in model 2 .

effect of the strain plus its potential interaction with $R$. insecticola. However, because strains with and without $R$. insecticola are similarly distributed in the phylogeny, potential interactions would only add noise to the analysis. This would decrease statistical power and thus should not create any false positives.

In the Spiroplasma phylogeny, clade 3 appears to have a lower rate of molecular evolution than clades 1 and 2 . We used the local-clock permutation test developed by Lanfear (2010) to assess whether this difference was significant. This test is independent of the above mentioned experiments and only concerns the molecular phylogeny. It uses the ratio between the likelihood of two models that are fitted to the phylogeny and its underlying sequences (GenBank IDs MG288511 to MG288588). The first model assumes a strict clock, meaning that all strains are evolving equally fast, while in the second model (local clocks), strains of clade 3 are allowed to evolve at a different rate than other strains. The $P$-value is obtained by comparing the observed ratio between the likelihoods of the two models to the null distribution of this ratio, which is estimated by refitting the strict and the local clock models to 10,000 permutations of the sequences. This test has been shown to be more conservative than the usual likelihood ratio-test (Lanfear 2010). The local clocks model applied to the real data was also used to estimate the effect size of the difference of rates of molecular evolution.

\section{Results}

\section{EXPERIMENT 1: EFFECT OF SPIROPLASMA ON A. ervi PARASITISM}

The "Regiella" model detected highly significant variation among wasp lines in the proportion of aphids that were mummified (i.e., parasitized successfully), but no overall effects of the presence of either Regiella or Spiroplasma (Table 1). The "Spiroplasma" model also recovered the strong differences among wasp lines, with line B being the most and line D the least virulent line (Fig. 2), as well as significant variation among aphid sub-lines, also in interaction with the wasp line (Table 1). Separate analyses for each wasp line showed that this was mostly due to variation in susceptibility to the most virulent wasp line B (Table 1), for which the presence of Spiroplasma strains S227 and S385(+Ri) reduced parasitism significantly (Table S3). In the case of wasp line K, aphids infected with strain S161 were more likely to be successfully parasitized than the uninfected control sub-line (Table S3). Wasp lines also differed in proportion emerged, line $\mathrm{K}$ having the highest and line D the lowest emergence rate. However, this difference was detected by model "Spiroplasma" but not by model "Regiella," likely because of the higher complexity of the latter model.

\section{EXPERIMENT 2: FITNESS COST OF SPIROPLASMA}

The overall effect of the symbionts Spiroplasma and R. insecticola on the fitness of their host is summarized by the intrinsic growth 

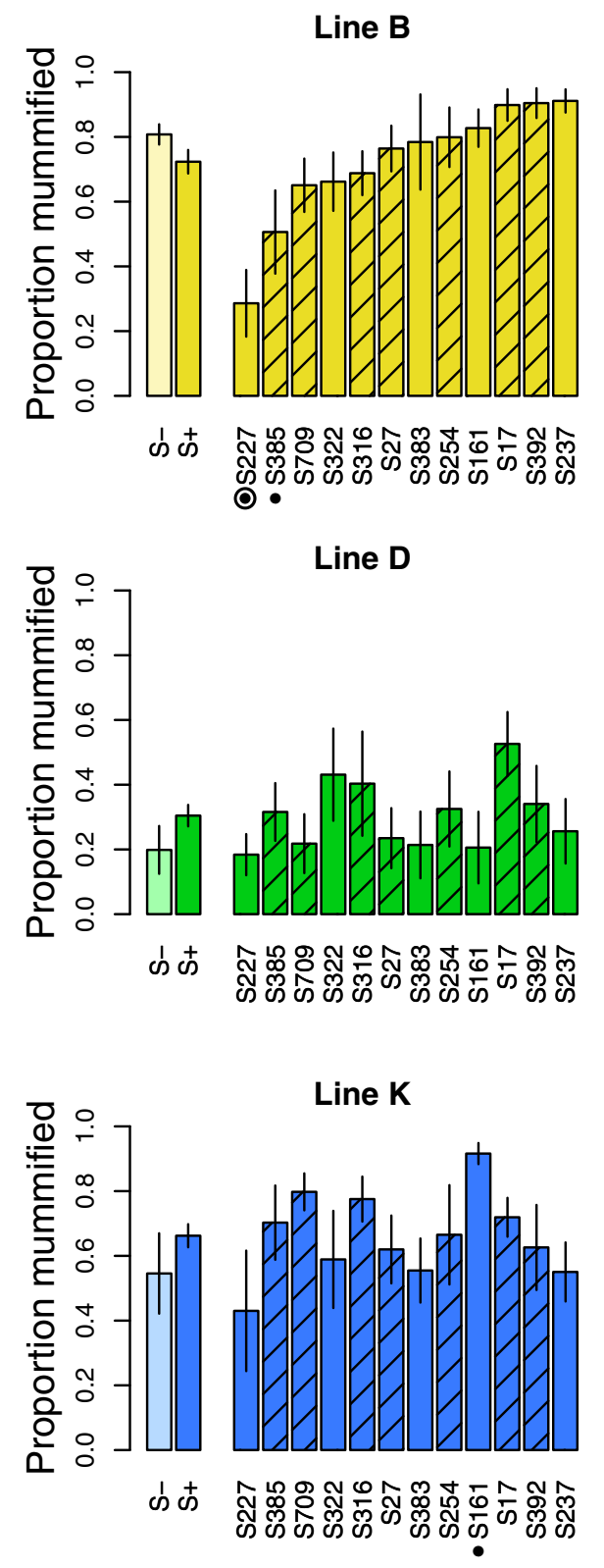

Figure 2. Mummification rates. Mean proportion of nymphs mummified ( \pm S.E. indicated with error bars) for the three wasp lines and each aphid sub-line. On each panel, $\mathbf{S}$ - corresponds to the uninfected subline, and $\mathrm{S}+$ to the mean of all Spiroplasma infected sub-lines. Error bars indicate the standard error. Sub-lines also containing $R$. insecticola are hatched. The significance of the comparisons between the Spiroplasma infected sub-lines and the uninfected control sub-lines performed from the 'Spiroplasma' models is indicated below the strains names ( $\bullet$ : only significant before adjusting for multiple comparisons; $\odot$ : still significant after adjusting for multiple comparisons).

rate. We repeat the caveat that the interpretation of these effects hinges on strong assumptions, namely that the average effect of Spiroplasma strains that are alone is comparable to that of strains that are sharing their host with $R$. insecticola, and that there are no interactive effects of Spiroplasma and $R$. insecticola on aphid phenotypes. Under these-admittedly untested-assumptions, it appears that Spiroplasma reduced the intrinsic growth rate significantly while $R$. insecticola increased it or at least counteracted the negative effect of Spiroplasma (Table 2 and Fig. 3A). Correcting for the estimated effect of $R$. insecticola, the "Spiroplasma" model shows that all Spiroplasma strains except S322, S383, and S237 decreased the intrinsic growth rate significantly. This was still significant for more than half of the strains after correcting for multiple testing (Table S3).

Infection by $R$. insecticola did not affect aphid lifespan, but all Spiroplasma-infected sub-lines had shorter lifespans than the Spiroplasma-free sub-line, on average by about eight days (Table 2, Fig. 3B). Only the effect of strains S27 and S385(+Ri) on host survival was no longer significant after accounting for multiple testing (Table S3). Spiroplasma also reduced lifetime reproduction while $R$. insecticola-with the caveat mentioned above-appeared to increase it or at least to counteract the negative effect of Spiroplasma (Table 2 and Fig. 3C). Neither infection with $R$. insecticola nor infection with Spiroplasma had a significant overall effect on the mean reproductive age of the aphid host (Tables 2 and S3).

\section{EXPERIMENT 3: VARIATION IN SPIROPLASMA DENSITY}

Infection by $R$. insecticola did not have any detectable effect on \#Spiroplasma dnaA, \#aphid EF1a, or their ratio in either 10- or 20-day-old aphids (Table 3). The \#aphid EF1a did not change significantly from age 10 to 20, but \#Spiroplasma dnaA increased strongly (Table 3, Fig. 4B and C), on average by a factor of 4.86, which corresponds to an average doubling time of 4.38 days for Spiroplasma. Accordingly, the ratio of Spiroplasma to aphid gene copies increased as well and reached very high values (approximately 40-130) in 20-day-old aphids. There was substantial variation in the densities and growth achieved by different Spiroplasma strains, reflected in the highly significant sub-line and age $\times$ sub-line effects on \#Spiroplasma dnaA (Table 3 ). This variation appeared to have a limited effect on aphid gene copy number, as the differences among sub-lines for \#aphid EF1a were not statistically significant $(P=0.08$, Table 3$)$. Spiroplasma strain S227 was notable, however, because this sub-line showed very low \#aphid EF1a in 10-day-old aphids, resulting in a high ratio of \#Spiroplasma dnaA/\#aphid EF1a (Fig. 4A). This is the sub-line that exhibited the lowest susceptibility to parasitoids but also high costs of infection by Spiroplasma (Figs. 2 and 3).

\section{PHYLOGENETIC SIGNAL IN SPIROPLASMA PHENOTYPES AND RATE OF MOLECULAR EVOLUTION}

The first two axes of the PCA that were tested for a phylogenetic signal summarized $57.03 \%$ of the phenotypic variation in the 12 Spiroplasma-infected pea aphid sub-lines. The first axis 
Table 2. Analyses of the fitness costs of Spiroplasma.

\begin{tabular}{|c|c|c|c|c|c|c|}
\hline \multirow{2}{*}{$\begin{array}{l}\text { Explained variable } \\
\text { Lifetime reproduction }\end{array}$} & \multicolumn{2}{|c|}{ Model } & \multirow{2}{*}{$\begin{array}{l}\text { Effect } \\
\text { Spiro. }(0 / 1)\end{array}$} & \multirow{2}{*}{ 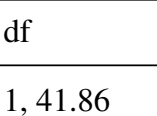 } & \multirow{2}{*}{$\frac{\mathrm{F}\left(\text { or } \chi^{2}\right)^{\#}}{9.27}$} & \multirow{2}{*}{$\frac{\text { p-value }}{0.004^{* *}}$} \\
\hline & 1 & "Regiella" & & & & \\
\hline & & & Regi. $(0 / 1)$ & $1,10.06$ & 6.61 & $0.028^{*}$ \\
\hline & 2 & "Spiroplasma" & Sub-line & $12,90.21$ & 5.46 & $<0.001^{* * *}$ \\
\hline \multirow[t]{3}{*}{ Lifespan } & 3 & "Regiella" & Spiro. $(0 / 1)$ & $1,173.99$ & 11.46 & $<0.001^{* * *}$ \\
\hline & & & Regi. $(0 / 1)$ & $1,173.99$ & 0.73 & 0.392 \\
\hline & 4 & "Spiroplasma" & Sub-line & $12,172.99$ & 95.61 & $<0.001^{* * *}$ \\
\hline \multirow[t]{3}{*}{ Mean reproductive age } & 5 & "Regiella" & Spiro. $(0 / 1)$ & $1,81.66$ & 0.18 & 0.673 \\
\hline & & & Regi. $(0 / 1)$ & $1,10.07$ & 1.65 & 0.227 \\
\hline & 6 & "Spiroplasma" & Sub-line & $12,141.42$ & 4.58 & $<0.001^{* * *}$ \\
\hline \multirow[t]{3}{*}{ Intrinsic growth rate } & 7 & "Regiella" & Spiro. $(0 / 1)$ & $1,51.57$ & 8.84 & $0.004^{* *}$ \\
\hline & & & Regi. $(0 / 1)$ & $1,10.01$ & 10.61 & $0.009^{* *}$ \\
\hline & 8 & "Spiroplasma" & Sub-line & $12,85.97$ & 4.19 & $<0.001^{* * *}$ \\
\hline
\end{tabular}

Models $1,3,5$, and 7 estimate the effect of $R$. insecticola on four variables related to fitness. They were used to build the offsets correcting for the presence of $R$. insecticola in the other models estimating the effect of each Spiroplasma strain (models 2, 4, 6, and 8).

\#For lifespan, we used a Cox model, for which fixed effect were tested with LRT. In this case, we report the $\chi^{2}$ statistics.

Table 3. Analyses of the $q P C R$ estimates of the number of gene copies in 10 and 20 days old aphids.

\begin{tabular}{|c|c|c|c|c|c|c|c|}
\hline \multirow{2}{*}{$\begin{array}{l}\text { Explained variable } \\
\text { \#Spiro. dnaA/\#aphid EF1a }\end{array}$} & \multicolumn{2}{|c|}{ Model } & \multirow{2}{*}{$\begin{array}{l}\text { Aphid age } \\
\text { Both }\end{array}$} & \multirow{2}{*}{$\begin{array}{l}\text { Effect } \\
\text { Regi. }(0 / 1)\end{array}$} & \multirow{2}{*}{$\frac{d f}{1,10.01}$} & \multirow{2}{*}{$\begin{array}{l}F \\
121.34\end{array}$} & \multirow{2}{*}{$\begin{array}{r}P \text {-value } \\
0.765\end{array}$} \\
\hline & 1 & "Regiella" & & & & & \\
\hline & & & & Age & $1,106.05$ & 0.25 & $<0.001^{* * *}$ \\
\hline & & & & Age $\times$ Regi & $1,106.20$ & 2.12 & 0.148 \\
\hline & 2 & "Spiroplasma" & Both & Age & 1,96 & 314.95 & $<0.001^{* * *}$ \\
\hline & & & & Sub-line & 11,96 & 8.07 & $<0.001^{* * *}$ \\
\hline & & & & Age $\times$ Sub-line & 11,96 & 1.96 & $0.041^{*}$ \\
\hline & 3 & "Spiroplasma" & 10 days & Sub-line & 11,49 & 4.50 & $<0.001^{* * *}$ \\
\hline & 4 & "Spiroplasma" & 20 days & Sub-line & 11,47 & 5.38 & $<0.001^{* * *}$ \\
\hline \multirow[t]{8}{*}{ \#Spiro. dnaA } & 5 & "Regiella" & Both & Regi. $(0 / 1)$ & $1,10.01$ & 0.00 & 0.993 \\
\hline & & & & Age & $1,106.04$ & 262.80 & $<0.001^{* * *}$ \\
\hline & & & & Age $\times$ Regi . & $1,106.14$ & 0.00 & 0.985 \\
\hline & 6 & "Spiroplasma" & Both & Age & 1,96 & 728.20 & $<0.001^{* * *}$ \\
\hline & & & & Sub-line & 11,96 & 12.31 & $<0.001^{* * *}$ \\
\hline & & & & Age $\times$ Sub-line & 11,96 & 2.63 & $0.006^{* *}$ \\
\hline & 7 & "Spiroplasma" & 10 days & Sub-line & 11,49 & 5.66 & $<0.001^{* * *}$ \\
\hline & 8 & "Spiroplasma" & 20 days & Sub-line & 11,47 & 10.02 & $<0.001^{* * *}$ \\
\hline \multirow[t]{6}{*}{ \#aphid EF1a } & 9 & "Regiella" & Both & Regi. $(0 / 1)$ & $1,10.05$ & 1.31 & 0.278 \\
\hline & & & & Age & $1,106.22$ & 0.25 & 0.618 \\
\hline & & & & Age $\times$ Regi & $1,106.83$ & 4.00 & $0.048^{*}$ \\
\hline & 10 & "Spiroplasma" & Both & Age & 1,96 & 0.68 & 0.412 \\
\hline & & & & Sub-line & 11,96 & 1.70 & 0.084 . \\
\hline & & & & Age $\times$ Sub-line & 11,96 & 1.16 & 0.325 \\
\hline
\end{tabular}

Models 1, 5, and 9 estimate the effect of $R$. insecticola on \#Spiroplasma dnaA/\#aphid EF1a, \#Spiroplasma dnaA, and \#aphid EF1a. They were used to build the offsets correcting for the presence of $\boldsymbol{R}$. insecticola in the other models estimating the effect of each Spiroplasma strain (models 2,6 , and 10). When there was a significant interaction between aphid age and sub-line, separate models were fitted for each age group to investigate the interaction (models $3,4,7$, and 8).

mainly summarized the negative effect that Spiroplasma strains with a high density had on the lifespan of their host (Fig. 5A). This negative effect on lifespan had little effect on the aphids' intrinsic growth rate because this first axis has only a low correlation with the lifetime reproduction and a negative correlation with the mean reproductive age (i.e., short-lived aphids produced offspring earlier in life). The second axis encompasses variation related to aphid health and suitability for parasitoids. This axis was positively correlated to \#aphid EF1a, the aphid growth rate, the lifetime reproduction, and negatively correlated to the mean 


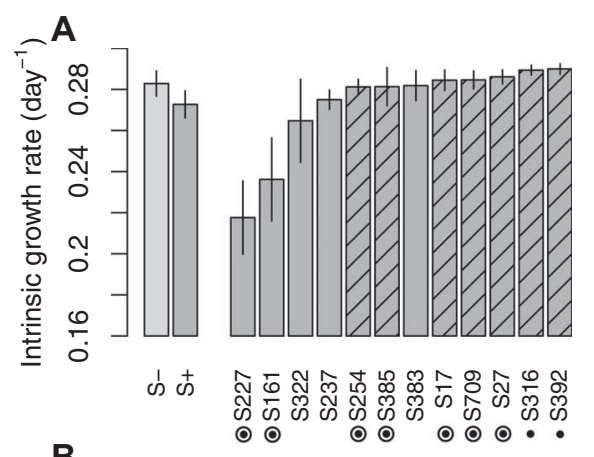

B

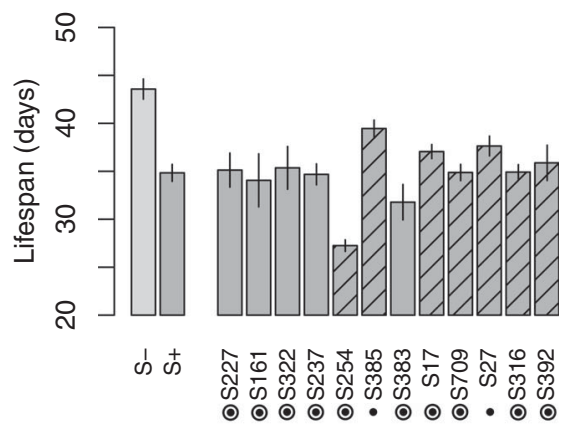

C
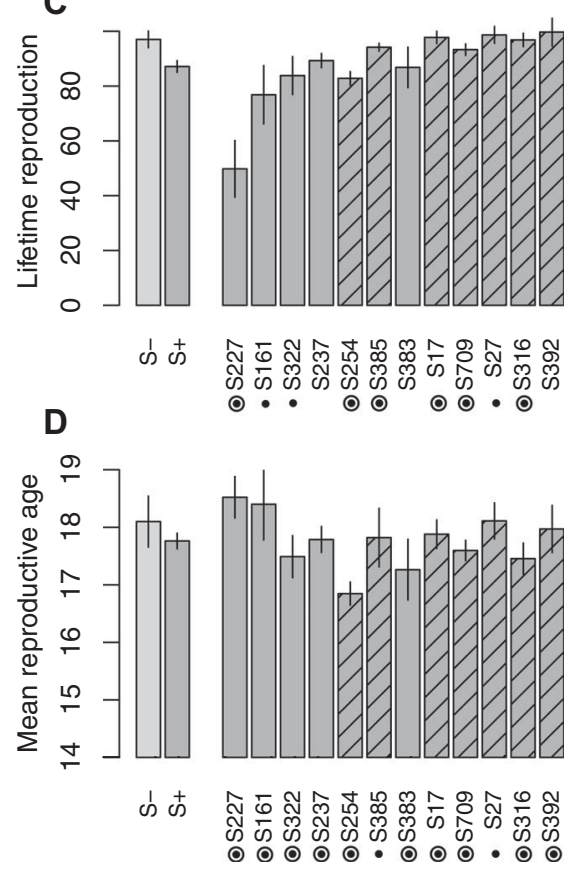

Figure 3. Spiroplasma effects on aphid fitness. Bar plots depicting the average intrinsic growth rate (A), lifespan (B), lifetime reproduction (C), and mean reproductive age (D) for all aphid sublines. On each panel, S- corresponds to the uninfected sub-line, and $\mathbf{S}+$ to the mean of all Spiroplasma infected sub-lines. Error bars indicate the standard error. Sub-lines also containing $R$. insecticola are hatched. The significance of the comparisons between the Spiroplasma infected sub-lines and the uninfected control sublines performed from the "Spiroplasma" models is indicated below the strains' names ( $\bullet$ : only significant before adjusting for multiple comparisons; $\odot$ : still significant after adjusting for multiple comparisons).
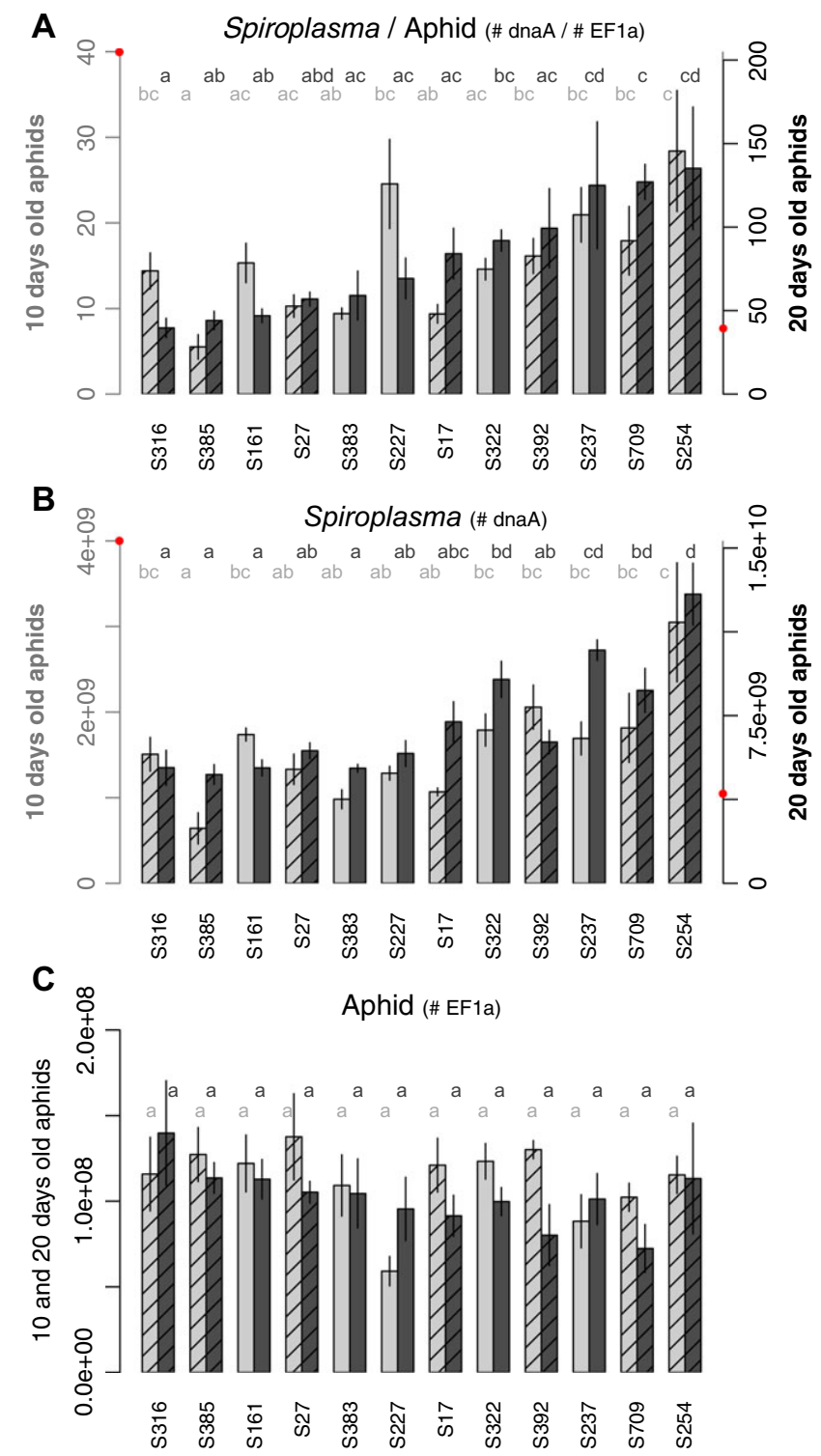

Figure 4. Spiroplasma density. The number of Spiroplasma gene copies \#per aphid gene copy (\#Spiroplasma dnaA/\# aphid EF1a) as well as the raw numbers of Spiroplasma and aphid gene copies per aphid individual (\# Spiroplasma dnaA and \# aphid EF1a) are shown on panels (A-C). Because \# Spiroplasma dnaA is much higher in 20 days old aphids (dark grey) than in 10 days old aphids (light grey), panels (A) and (B) have two $y$-axes with different scales. To help the comparison, red dots indicate the same values on the left and right axes. Different letters above bars indicate significant pairwise differences in Tukey-HSD tests. Error bars indicate the standard errors.

reproductive age. Sub-lines with a higher score on this axis (i.e., more fecund sub-lines) also showed higher rates of mummification by parasitoids and parasitoid emergence (Fig. 5B). The variation in the reproductive fitness of the sublines was not a function of Spiroplasma titers, as the variation in \#Spiroplasma dnaA was only weakly correlated with this axis. 
A
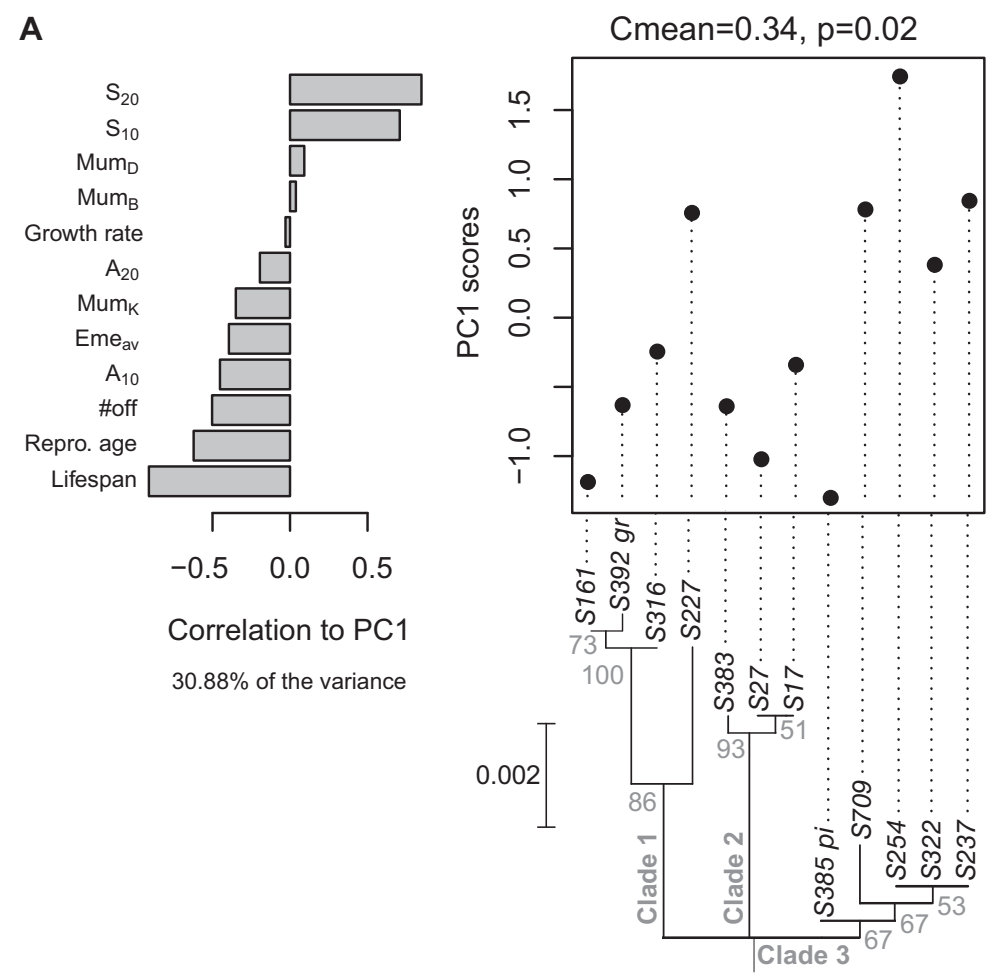

B

Correlation to PC1

$30.88 \%$ of the variance
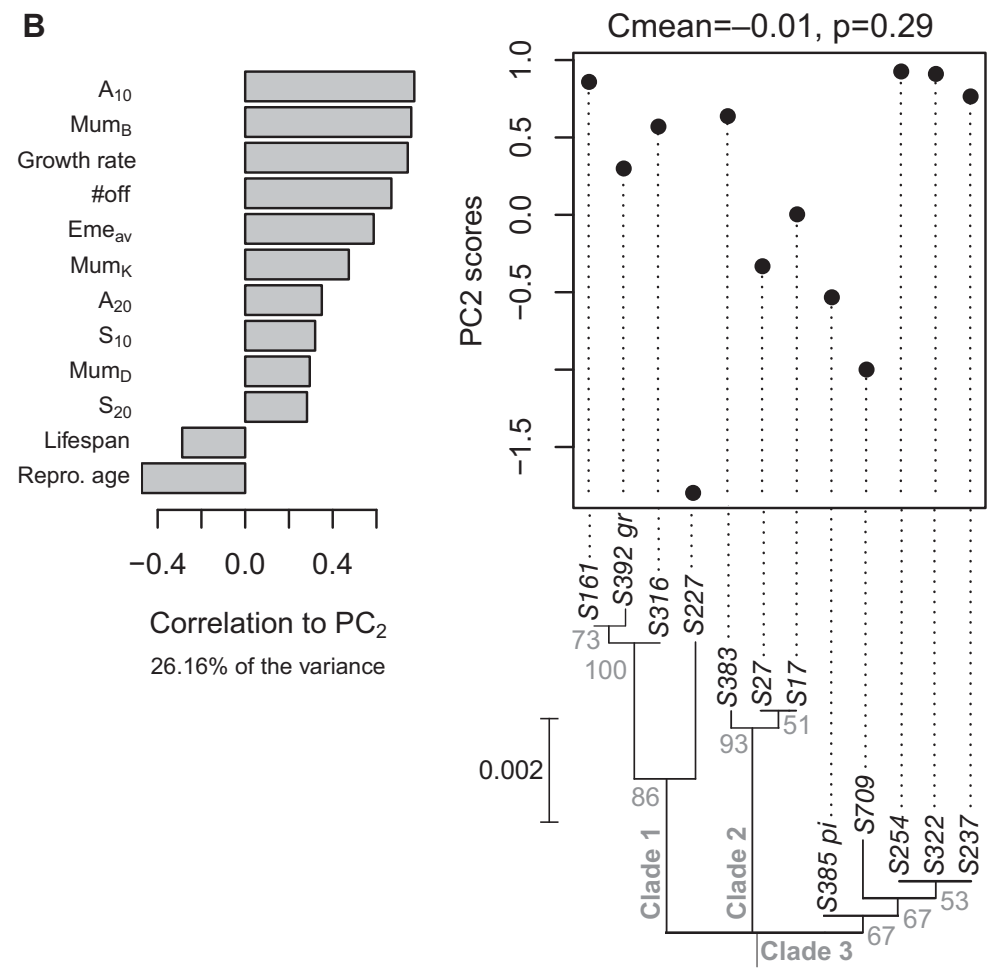

Figure 5. Phylogenetic signal in the Spiroplasma induced phenotypes. (A and B) Phylogenetic signal in principal components (PC) 1 and 2, summarizing the phenotypic variation induced by the different Spiroplasma strains. The PCA was done on the coefficients of the models fitted to the phenotypic traits of the aphids. On both panels, the horizontal bar plot indicates the correlation between the model coefficients that are the variables of the PCA and the corresponding PCA axis. This informs about the phenotypes of the aphids that are summarized by the PCA axis. Variable names were shortened: $S$ and A refer to \#Spiroplasma dnaA and \#aphid EF1a in 10 and 20 days old aphids; Mum and Eme refer to the mummification and emergences rates, respectively, in wasps B, D, and K and averaged (av) over the three wasp lines; \#off refers to lifetime reproduction. The plots on the right side give the score of each Spiroplasma strain on the PCA axes and its position in the phylogeny. The scale bar indicates the substitution rate. 
These two axes were used to investigate the correlation between the Spiroplasma-induced phenotypic variation and the Spiroplasma phylogeny using the Abouheif's $\mathrm{C}_{\text {mean }}$ statistic. Only the first axis was significantly correlated $\left(\mathrm{PC} 1 \mathrm{C}_{\text {mean }}=0.34, P=\right.$ 0.02 ; $\left.\mathrm{PC} 2: \mathrm{C}_{\text {mean }}=-0.01, P=0.29\right)$, with most strains of clade 3 having a high score on the first axis (Fig. 5A).

The local clock model estimated that the sequences of clade 3 are evolving 5.6 times more slowly than those of clades 1 and 2. The local-clock permutation test revealed that this difference was marginally significant $(P=0.043)$.

\section{Discussion}

In the absence of reproductive manipulation or frequent horizontal spread, heritable endosymbionts must provide a net fitness benefit to persist in host populations (Oliver et al. 2014). We investigated protection against the parasitoid wasp $A$. ervi as a potential benefit provided by 12 different strains of Spiroplasma in pea aphids, and we estimated their costs to the host in terms of life-history traits.

Evidence for protection was very limited and restricted to one of the three lines of A. ervi we used. Only Spiroplasma strains S227 and S385 reduced parasitism by the most virulent wasp line B significantly. In the case of S227, however, this was associated with very low reproductive fitness of the aphids in the absence of parasitoids, suggesting that S227-infected aphids were generally of poor health. On the other hand, when the aphids were exposed to wasp line K, one strain of Spiroplasma (S161) even seemed to represent a significant liability and made aphids more susceptible to parasitism. The effects of some Spiroplasma strains tended to be unequal across the three parasitoid lines, which resulted in a near-significant genotype-by-genotype interaction (Table 1). In principle, such interactions could contribute to the maintenance of strain diversity in parasites as well as symbionts (Kwiatkowski and Vorburger 2012; Ford et al. 2017; Vorburger and Perlman 2018), although their importance is questionable here, because the majority of Spiroplasma strains had no detectable effects on parasitism. We do not know why the three wasp lines varied so strongly in their parasitism efficacy. The conspicuously low success of line D could be related to the long time it has been reared in our laboratory at relatively small population size, which might have resulted in negative effects of inbreeding. The difference between the two commercially available lines may be related to their long-term rearing conditions in the production and/or their genetic background. Genetic variation in parasitism success is commonplace in parasitoids (Kraaijeveld and Godfray 1999; Colinet et al. 2010; Sandrock et al. 2010) and likely related to variation in the cocktail of virulence factors parasitoids employ. For example, parasitoid wasp venom is a major source of virulence factors and generally shows a high level of intraspecific variation (Colinet et al. 2013; Mathé-Hubert et al. 2015), also in A. ervi (Colinet et al. 2014). Interactions between parasitoid virulence factors and Spiroplasma in the aphid hosts could potentially explain the somewhat uneven effects of the different Spiroplasma strains in the three parasitoid treatments.

Even though we find little evidence for protection against $A$. ervi in the present study, it should be added that Spiroplasma may still reduce the risk of parasitism indirectly via a plantmediated effect, because A. ervi is more attracted to volatiles from plants infested by Spiroplasma-free aphids than from plants with Spiroplasma-infected aphids, as recently shown by Frago et al. (2017). Such an effect would have been missed by our non-choice assays.

Due to an unfortunate handling error in the preparation of our experimental lines, about half of the Spiroplasma strains shared their hosts with a coinfection of $R$. insecticola. However, the presence of $R$. insecticola did not have any detectable effects on susceptibility to A. ervi. This outcome is consistent with earlier studies that tested the same strain of $R$. insecticola deliberately and reported no significant effects on parasitism by A. ervi (Oliver et al. 2003; Hansen et al. 2012).

In contrast to the potential benefits we investigated, the costs of infection with Spiroplasma were rather clear. All strains curtailed aphid lifespan significantly, on average by more than 8 days. A reduction of host lifespan is also characteristic of Spiroplasma infection in Drosophila melanogaster (Herren et al. 2014). Because old aphids are less fecund than young adults (e.g., Zeng et al. 1993; Vorburger and Ramsauer 2008), and because offspring produced early in life are more important for a clone's growth rate than offspring produced late (Lenski and Service 1982), the strong negative effect on lifespan did not translate into equally strong effects on lifetime reproduction and the intrinsic rate of increase (Fig. 3). Nevertheless, two of the five sub-lines infected only by Spiroplasma showed significantly lower intrinsic rates of increase than the uninfected control. The sub-lines in which Spiroplasma co-occurred with $R$. insecticola showed similar trait values to the uninfected sub-line. This would suggest that the presence of $R$. insecticola counterbalanced the costs imposed by Spiroplasma. The "Regiella" models indeed showed a positive overall effect of $R$. insecticola on lifetime reproduction as well as the intrinsic rate of increase. This interpretation of the results is, however, conditional on the validity of the assumption that Spiroplasma strains associated with $R$. insecticola have the same average effect as those that are not. A positive effect of $R$. insecticola on host fitness has also been reported by Tsuchida et al. (2004) for pea aphids feeding on clover, but this does not seem to be a general property of this symbiont (Leonardo 2004; Ferrari et al. 2007), and other studies reported negative fitness effects of this symbiont, for example, in the grain aphid, Sitobion avenae (Wang et al. 2016; Luo et al. 2017). Nonetheless, it has been observed before that one heritable 
endosymbiont can compensate the costs imposed by another. Doremus and Oliver (2017) found that the large costs associated with the possession of X-type endosymbionts in pea aphids were ameliorated by coinfection with $H$. defens $a$. When the influence of $R$. insecticola was corrected for statistically in the present data, the majority of Spiroplasma strains were inferred to reduce lifetime reproduction and intrinsic rate of increase significantly (Table S3). Thus we conclude that under laboratory conditions and in the absence of any other selective forces, infection with Spiroplasma generally has a negative effect on pea aphid reproductive fitness.

To some extent, the Spiroplasma-induced fitness costs were related to the symbionts' densities in the host. The Spiroplasma titers in pea aphids increased strongly from the age of 10 days to the age of 20 days, suggesting that the host has limited control over the proliferation of Spiroplasma. This is also observed in D. melanogaster, and it may be related to the fact that cell wall-less bacteria like Spiroplasma can escape the attention of the insect immune system (Herren and Lemaitre 2011; Herren et al. 2014). However, not all strains were equally prolific. Spiroplasma titers varied substantially among aphid sub-lines, and there was no indication that they were influenced by coinfecting $R$. insecticola. The links among the estimated effects of the different Spiroplasma strains on the various traits we measured was investigated with a PCA on the coefficients estimated by the models analyzing these traits. In this PCA, the first PC was chiefly associated with high Spiroplasma densities and short aphid lifespan, providing correlative evidence that higher Spiroplasma titers are more harmful to the host. Interestingly, there was a weak but significant phylogenetic signal in the variation along this axis (Fig. 5). This was mostly because strains from clade 3 showed higher scores for PC1 on average, i.e., these strains achieved higher densities and tended to be associated with shorter host lifespans. High Spiroplasma densities have also been shown to curtail host lifespan in flies (Herren and Lemaitre 2011). Clade 3 also exhibited a lower rate of molecular evolution than the other two clades, and it is tempting to speculate about a causal link with the seemingly more parasitic lifestyle of these Spiroplasma strains. Endosymbiotic bacteria generally exhibit increased rates of sequence evolution than their free-living relatives, which is attributed to the lower effective population size that comes with maternal transmission and the associated bottlenecks between host generations (Moran 1996; Woolfit and Bromham 2003; Boscaro et al. 2013). Longterm vertical transmission is also expected to reduce the costs that symbionts impose on their hosts. Endosymbionts are thus a good model of how organisms can move along the parasitismmutualism continuum (Ewald 1987; King 2019). It might seem that Spiroplasma strains from clade 3 occupy a space further toward the parasitic end of this continuum than the other clades. Whether this reflects a shorter association with the host, which would be consistent with the slower rate of molecular evolution, or whether other selective forces have created this situation, is currently unknown. In this context it could be relevant that the different clades of Spiroplasma tend to be associated with different communities of co-infecting symbionts in natural populations of pea aphids. For example, clade 3 Spiroplasma are positively associated in the field with the X-type symbiont and negatively with $H$. defensa, while those from clade 2 tend to be positively associated with Rickettsia, and this seems to be unrelated to the host plants from which pea aphids were collected (Mathé-Hubert et al. 2018). Regular coinfections with other bacteria certainly have the potential to affect the evolution of endosymbionts and possibly their virulence (Vorburger and Perlman 2018). Interactions with other species of endosymbiotic bacteria thus clearly deserve attention to better understand Spiroplasma's influence on host ecology and evolution.

In conclusion, our experiment showed that infections with various strains of the heritable endosymbiont Spiroplasma are rather costly to their pea aphid hosts, and that protection against A. ervi is unlikely to compensate for these costs. We tested for protection against $A$. ervi because it is the pea aphid's most common parasitoid, but multiple parasitoids include pea aphids in their host range and we cannot exclude that Spiroplasma may be protective against other species. Already demonstrated is a protective effect of certain strains of Spiroplasma, including strain S161 used here, against the entomopathogenic fungus Pandora neoaphidis (Łukasik et al. 2013), but also this is not a general property of all Spiroplasma found in pea aphids. Once a symbiont has evolved maternal transmission, it is under strong selection to keep its host alive until reproduction. This can be achieved via protection against natural enemies or by providing other ecological benefits, for example, by increasing tolerance to abiotic stressors (Oliver et al. 2010). The specific mechanisms may well vary among different strains of the same symbiont species, and with the high diversity of strains structured into at least three clades, Spiroplasma of pea aphids is an attractive model to investigate this variation further.

\section{AUTHOR CONTRIBUTIONS}

H.K., P.G., C.V., and H.M.H. performed the experiments; P.G., H.M.H., and H.K. carried out the molecular analysis; H.M.H. was responsible for the data analysis; H.M.H., H.K., and C.V. wrote the paper.

\section{ACKNOWLEDGMENTS}

The authors are very grateful to Paula Rodriguez for her help with insect rearing. The authors also thank Ailsa McLean for sharing several Spiroplasma-infected aphid lines. The handling editor and the reviewers provided very helpful comments to improve the manuscript. This work was supported by a Sinergia grant from the Swiss National Science Foundation (grant nr. CRSII3 154396 to C.V.). 


\section{DATA ARCHIVING}

The DNA sequences used in this study are available in Genbank (accession numbers: MG288511 to MG288588). The main dataset is deposited in the Dryad Digital Repository: Literature cited

\section{LITERATURE CITED}

Asplen, M. K., N. Bano, C. M. Brady, N. Desneux, K. R. Hopper, C. Malouines, K. M. Oliver, J. A. White, and G. E. Heimpel. 2014. Specialisation of bacterial endosymbionts that protect aphids from parasitoids. Ecol. Entomol. 39:736-739.

Ballinger, M. J., and S. J. Perlman. 2018. The defensive spiroplasma. Curr. Opin. Insect Sci. 32:1-6.

Bates, D., M. Maechler, B. Bolker, and S. Walker. 2015. 1me4: Linear mixedeffects models using Eigen and S4. R package version 1.1-8.

Bian, G., Y. Xu, P. Lu, Y. Xie, and Z. Xi. 2010. The endosymbiotic bacterium Wolbachia induces resistance to dengue virus in Aedes aegypti. PLoS One 6:e1000833.

Birch, L. C. 1948. The intrinsic rate of natural increase of an insect population. J. Anim. Ecol. 17:15-26.

Boscaro, V., M. Felletti, C. Vannini, M. S. Ackerman, P. S. G. Chain, S. Malfatti, L. M. Vergez, M. Shin, T. G. Doak, M. Lynch, et al. 2013. Polynucleobacternecessarius, a model for genome reduction in both free-living and symbiotic bacteria. Proc. Natl. Acad. Sci. USA 110:18590-5.

Bové, J. M., J. Renaudin, C. Saillard, X. Foissac, and M. Garnier. 2003. Spiroplasma citri, a plant pathogenic mollicute: relationships with its two hosts, the plant and the leafhopper vector. Annu. Rev. Phytopathol. 41:483-500.

Caillaud, M. C., M. Boutin, C. Braendle, and J.-C. Simon. 2002. A sex-linked locus controls wing polymorphism in males of the pea aphid, Acyrthosiphon pisum (Harris). Heredity (Edinb). 89:346352.

Cayetano, L., and C. Vorburger. 2013. Genotype-by-genotype specificity remains robust to average temperature variation in an aphid/endosymbiont/ parasitoid system. J. Evol. Biol. 26:1603-10.

Cayetano, L., and C. Vorburger. 2014. Symbiont-conferred protection against Hymenopteran parasitoids in aphids: how general is it? Ecol. Entomol. 40:1-9.

Chen, X., S. Li, and S. Aksoy. 1999. Concordant evolution of a symbiont with its host insect species: molecular phylogeny of genus Glossina and its bacteriome-associated endosymbiont, Wigglesworthia glossinidia. J. Mol. Evol. 48:49-58.

Chiel, E., Y. Gottlieb, N. Katzir, M. Inbar, and M. Ghanim. 2007. Biotypedependent secondary symbiont communities in sympatric populations of Bemisia tabaci. Bull. Entomol. Res. 97:407-413.

Clark, T. B., R. F. Whitcomb, J. G. Tully, C. Mouches, C. Saillard, J. M. Bové, H. Wroblewski, P. Carle, D. L. Rose, R. B. Henegar, et al. 1985. Spiroplasma melliferum, a new species from the Honeybee (Apis mellifera). Int. J. Syst. Bacteriol. 35:296-308.

Colinet, D., C. Anselme, E. Deleury, D. Mancini, J. Poulain, C. Azéma-Dossat, M. Belghazi, S. Tares, F. Pennacchio, M. Poirié, et al. 2014. Identification of the main venom protein components of Aphidius ervi, a parasitoid wasp of the aphid model Acyrthosiphon pisum. BMC Genomics 15: $1-14$.

Colinet, D., H. Mathé-Hubert, R. Allemand, J. L. J. L. Gatti, and M. Poirié. 2013. Variability of venom components in immune suppressive parasitoid wasps: from a phylogenetic to a population approach. J. Insect Physiol. 59:205-12.

Colinet, D., A. Schmitz, D. Cazes, and J. Gatti. 2010. The origin of intraspecific variation of virulence in an eukaryotic immune suppressive parasite. PLoS Pathog. 6:1-11.
Wang, D., X. Shi, P. Dai, D. Liu, X. Dai, Z. Shang, Z. Ge, and X. Meng. 2016. Comparison of fitness traits and their plasticity on multiple plants for Sitobion avenae infected and cured of a secondary endosymbiont. Sci. Rep. 6:23177.

Doremus, M. R., and K. M. Oliver. 2017. Aphid heritable symbiont exploits defensive mutualism. Appl. Environ. Microbiol. 83:1-15.

Douglas, A. E. 1998. Nutritional interactions in insect-microbial symbioses: aphids and their symbiotic bacteria buchnera. Annu. Rev. Entomol. 43:17-37.

Duron, O., D. Bouchon, S. Boutin, L. Bellamy, L. Zhou, J. Engelstadter, and G. D. Hurst. 2008. The diversity of reproductive parasites among arthropods: Wolbachia do not walk alone. BMC Biol. $6: 27$.

Ewald, P. W. 1987. Transmission modes and evolution of the parasitismmutualism continuum. Ann. N. Y. Acad. Sci. 503:295-306.

Ferrari, J., C. L. Scarborough, and H. C. J. Godfray. 2007. Genetic variation in the effect of a facultative symbiont on host-plant use by pea aphids. Oecologia 153:323-329.

Ferrari, J., J. a West, S. Via, and H. C. J. Godfray. 2012. Population genetic structure and secondary symbionts in host-associated populations of the pea aphid complex. Evolution (N. Y). 66:375-390.

Ford, S. A., D. Williams, S. Paterson, and K. C. King. 2017. Co-evolutionary dynamics between a defensive microbe and a pathogen driven by fluctuating selection. Mol. Ecol. 26:1778-1789.

Frago, E., M. Mala, B. T. Weldegergis, C. Yang, A. McLean, H. C. J. Godfray, R. Gols, and M. Dicke. 2017. Symbionts protect aphids from parasitic wasps by attenuating herbivore-induced plant volatiles. Nat. Commun. $8: 1-9$.

Gasparich, G. E., R. F. Whitcomb, D. Dodge, F. E. French, J. Glass, and D. L. Williamson. 2004. The genus Spiroplasma and its non-helical descendants: Phylogenetic classification, correlation with phenotype and roots of the Mycoplasma mycoides clade. Int. J. Syst. Evol. Microbiol. 54:893-918.

Halekoh, U., and S. Højsgaard. 2014. A Kenward-Roger approximation and parametric bootstrap methods for tests in linear mixed models - the R package pbkrtest. J. Stat. Softw. 59:1-32.

Hansen, A. K., C. Vorburger, and N. A. Moran. 2012. Genomic basis of endosymbiont-conferred protection against an insect parasitoid. Genome Res. 22:106-114.

Harrell, F. E. 2017. rms: Regression modeling strategies. R package version $5: 1-1$.

Harrison, X. A. 2015. A comparison of observation-level random effect and Beta-Binomial models for modelling overdispersion in Binomial data in ecology \& evolution. PeerJ 3:e1114.

Heath, K. D., and J. R. Stinchcombe. 2014. Explaining mutualism variation: a new evolutionary paradox? Evolution 68:309-317.

Hedges, L. M., J. C. Brownlie, S. L. O’Neill, and K. N. Johnson. 2008. Wolbachia and virus protection in insects. Science 322:702.

Henry, L. M., J. Peccoud, J. C. Simon, J. D. Hadfield, M. J. C. Maiden, J. Ferrari, and H. C. J. Godfray. 2013. Horizontally transmitted symbionts and host colonization of ecological niches. Curr. Biol. 23:1713-1717.

Herren, J. K., and B. Lemaitre. 2011. Spiroplasma and host immunity: activation of humoral immune responses increases endosymbiont load and susceptibility to certain Gram-negative bacterial pathogens in Drosophila melanogaster. Cell. Microbiol. 13:1385-1396.

Herren, J. K., J. C. Paredes, F. Schüpfer, K. Arafah, P. Bulet, B. Lemaitre, L. Epfl, P. B. Archamps, and S. Julien. 2014. Insect endosymbiont proliferation is limited by lipid availability. Elife 3:e02964.

Hilgenboecker, K., P. Hammerstein, P. Schlattmann, A. Telschow, and J. H. Werren. 2008. How many species are infected with Wolbachia? A statistical analysis of current data. FEMS Microbiol. Lett. 281:215-20. 
Hothorn, T., F. Bretz, and P. Westfall. 2008. Simultaneous inference in genera parametric models. Biometric. J. 50:346-363.

Hutchinson, M. K., and M. C. Holtman. 2005. Focus on research methods analysis of count data using Poisson regression ${ }^{*}$. Res. Nurs. Health 28:408-418.

Jaenike, J., R. Unckless, and S. Cockburn. 2010. Adaptation via symbiosis: recent spread of a Drosophila defensive symbiont. Science (80-.). 329:212-215.

Keck, F., F. Rimet, A. Bouchez, and A. Franc. 2016. phylosignal: an R package to measure, test, and explore the phylogenetic signal. Ecol. Evol. 6:27742780 .

King, K. C. 2019. Defensive symbionts. Curr. Biol. 29:R78-R80.

Kraaijeveld, A. R., and H. C. J. Godfray. 1999. Geographic patterns in the evolution of resistance and virulence in drosophila and its parasitoids. Am. Nat. 153:61-74.

Kwiatkowski, M., J. Engelstädter, and C. Vorburger. 2012. On genetic specificity in symbiont-mediated host-parasite coevolution. PLoS Comput. Biol. 8:e1002633.

Kwiatkowski, M., and C. Vorburger. 2012. Modeling the ecology of symbiontmediated protection against parasites. Am. Nat. 179:595-605.

Lanfear, R. 2010. The local-clock permutation test: a simple test to compare rates of molecular evolution on phylogenetic trees. Evolution 65:606611.

Lenski, R. E., and P. M. Service. 1982. The statistical analysis of population growth rates calculated from schedules of survivorship and fecunidity. Ecology 63:655-662.

Leonardo, T. E. 2004. Removal of a specialization-associated symbiont does not affect aphid fitness. Ecol. Lett. 7:461-468.

Leybourne, D. J., J. I. Bos, T. A. Valentine, and A. J. Karley. 2018. The price of protection: a defensive endosymbiont impairs nymph growth in the bird cherry-oat aphid, Rhopalosiphum padi. Insect Science.

Łukasik, P., H. Guo, M. Van Asch, J. Ferrari, and H. C. J. Godfray. 2013. Protection against a fungal pathogen conferred by the aphid facultative endosymbionts Rickettsia and Spiroplasma is expressed in multiple host genotypes and species and is not influenced by co-infection with another symbiont. J. Evol. Biol. 26:2654-2661.

Luo, C., K. Luo, L. Meng, B. Wan, H. Zhao, and Z. Hu. 2017. Ecological impact of a secondary bacterial symbiont on the clones of Sitobion avenae (Fabricius) (Hemiptera: Aphididae). Nat. Publ. Gr. 7:1-8.

Martinez, A. J., K. L. Kim, J. P. Harmon, and K. M. Oliver. 2016. Specificity of multi-modal aphid defenses against two rival parasitoids. PLoS One 11:e0154670.

Mathé-Hubert, H., J.-L. Gatti, D. Colinet, M. Poirié, and T. Malausa. 2015. Statistical analysis of the individual variability of $1 \mathrm{D}$ protein profiles as a tool in ecology: an application to parasitoid venom. Mol. Ecol. Resour. 15:1120-1132.

Mathé-Hubert, H., H. Kaech, C. Hertaeg, and C. Vorburger. 2018. Nonrandom associations of maternally transmitted symbionts in insects: The roles of drift versus co-transmission and selection. bioRxiv 1-30. https://doi.org/10.1101/364653.

McLean, A. H. C. 2019. Cascading effects of defensive endosymbionts. Curr. Opin. Insect Sci. 32:42-46.

Mclean, A. H. C., and H. C. J. Godfray. 2015. Evidence for specificity in symbiont- conferred protection against parasitoids. Proc. R. Soc. B. 282:1-8.

McLean, A. H. C., and H. C. J. Godfray. 2017. The outcome of competition between two parasitoid species is influenced by a facultative symbiont of their aphid host. Funct. Ecol. 31:927-933.

McLean, A. H. C., M. van Asch, J. Ferrari, and H. C. J. Godfray. 2011. Effects of bacterial secondary symbionts on host plant use in pea aphids. Proc. Biol. Sci. 278:760-766.
Moran, N. A. 1996. Accelerated evolution and Muller's rachet in endosymbiotic bacteria. Proc. Natl. Acad. Sci. USA 93:2873-2878.

Moran, N. a, J. P. McCutcheon, and A. Nakabachi. 2008. Genomics and evolution of heritable bacterial symbionts. Annu. Rev. Genet. 42:165190.

Moran, N., and J. Russell. 2005. Evolutionary relationships of three new species of enterobacteriaceae living as symbionts of aphids and other insects. Appl. Environ. Microbiol. 71:3302-3310.

Münkemüller, T., S. Lavergne, B. Bzeznik, S. Dray, T. Jombart, K. Schiffers, and W. Thuiller. 2012. How to measure and test phylogenetic signal. Methods Ecol. Evol. 3:743-756.

Oliver, K. M., J. Campos, N. a Moran, and M. S. Hunter. 2008. Population dynamics of defensive symbionts in aphids. Proc. Biol. Sci. 275:293299.

Oliver, K. M., P. H. Degnan, G. R. Burke, and N. a Moran. 2010. Facultative symbionts in aphids and the horizontal transfer of ecologically important traits. Annu. Rev. Entomol. 55:247-266.

Oliver, K. M., and N. A. Moran. 2009. Defensive symbionts in aphids and other insects. In: Defensive mutualism in microbial symbiosis, eds., White J. F. and Torres M. S.. Taylor \& Francis, Abingdon, U.K., pp. 129-147.

Oliver, K. M., J. A. Russell, N. A. Moran, and M. S. Hunter. 2003. Facultative bacterial symbionts in aphids confer resistance to parasitic wasps. Proc. Natl. Acad. Sci. USA. 100:1803-7.

Oliver, K. M., A. H. Smith, and J. A. Russell. 2014. Defensive symbiosis in the real world-advancing ecological studies of heritable, protective bacteria in aphids and beyond. Funct. Ecol. 28:341-355.

Paredes, J. C., J. K. Herren, F. Schüpfer, and B. Lemaitre. 2016. The role of lipid competition for endosymbiont-mediated protection against parasitoid wasps in Drosophila. MBio 7:e01006-16.

Parker, B. J., J. Hrcek, A. H. C. McLean, and H. C. J. Godfray. 2017. Genotype specificity among hosts, pathogens, and beneficial microbes influences the strength of symbiont-mediated protection. Evolution 71:12221231.

Raychoudhury, R., L. Baldo, D. C. S. G. Oliveira, and J. H. Werren. 2009. Modes of acquisition of Wolbachia: horizontal transfer, hybrid introgression, and codivergence in the Nasonia species complex. Evolution 63:165-183.

Russell, J. a., S. Weldon, A. H. Smith, K. L. Kim, Y. Hu, P. Łukasik, S. Doll, I. Anastopoulos, M. Novin, and K. M. Oliver. 2013. Uncovering symbiont-driven genetic diversity across North American pea aphids. Mol. Ecol. 22:2045-2059.

Sandrock, C., A. Gouskov, and C. Vorburger. 2010. Ample genetic variation but no evidence for genotype specificity in an all-parthenogenetic hostparasitoid interaction. J. Evol. Biol. 23:578-585.

Scarborough, C. L., J. Ferrari, and H. C. J. Godfray. 2005. Aphid protected from pathogen by endosymbiont. Science 310:1781.

Schmid, M., R. Sieber, Y.-S. S. Zimmermann, and C. Vorburger. 2012. Development, specificity and sublethal effects of symbiontconferred resistance to parasitoids in aphids. Funct. Ecol. 26:207215.

Simon, J. C., S. Boutin, T. Tsuchida, R. Koga, J. F. Gallic, A. Frantz, Y. Outreman, and T. Fukatsu. 2011. Facultative symbiont infections affect aphid reproduction. PLoS One 6:e21831.

Smyth, G. K. 2005. Limma: linear models for microarray data. In: Bioinformatics and computational biology solutions using $\mathrm{R}$ and bioconductor, eds., Gentleman R., Carey V., Huber W., Dudoit S., and Irizarry R.. Springer, New York, NY, pp. 397-420.

Teixeira, L., A. Ferreira, and M. Ashburner. 2008. The bacterial symbiont Wolbachia induces resistance to RNA viral infections in Drosophila melanogaster. PLoS Biol. 6:e1000002. 
The International Aphid Genomics Consortium. 2010. Genome sequence of the pea aphid Acyrthosiphon pisum. PLoS Biol. 8:e1000313.

Therneau, T. M. 2015a. coxme: mixed effects cox models. 1-14.

Therneau, T. M. 2015b. A package for survival analysis in S. version 2.38

Tsuchida, T., R. Koga, and T. Fukatsu. 2004. Host plant specialization governed by facultative symbiont. Science 303:1989.

Vorburger, C. 2014. The evolutionary ecology of symbiont-conferred resistance to parasitoids in aphids. Insect Sci. 21:251-64.

Vorburger, C., L. Gehrer, and P. Rodriguez. 2010. A strain of the bacterial symbiont Regiella insecticola protects aphids against parasitoids. Biol. Lett. 6:109-111.

Vorburger, C., and A. Gouskov. 2011. Only helpful when required: A longevity cost of harbouring defensive symbionts. J. Evol. Biol. 24:16111617.

Vorburger, C., and S. J. Perlman. 2018. The role of defensive symbionts in host-parasite coevolution. Biol. Rev., https://doi.org/10.1111/brv.12417.

Vorburger, C., and N. Ramsauer. 2008. Genetic variation and covariation of aphid life-history traits across unrelated host plants. Bull. Entomol. Res. 98:543-553.

Vorburger, C., and R. Rouchet. 2016. Are aphid parasitoids locally adapted to the prevalence of defensive symbionts in their hosts? BMC Evol. Biol. 16:271.

Wang, W., W. Gu, Z. Ding, Y. Ren, J. Chen, and Y. Hou. 2005. A novel Spiroplasma pathogen causing systemic infection in the crayfish Procambarus clarkii (Crustacea: Decapod), in China. FEMS Microbiol. Lett. 249:131-137.

Warton, D. I., and F. K. C. Hui. 2011. The arcsine is asinine: the analysis of proportions in ecology. Ecology 92:3-10.
Watts, T., T. S. Haselkorn, N. A. Moran, and T. A. Markow. 2009. Variable incidence of Spiroplasma infections in natural populations of Drosophila species. PLoS One 4:e5703.

Wernegreen, J. J. 2002. Genome evolution in bacterial endosymbionts of insects. Nat. Rev. Genet. 3:850-861.

Werren, J. H., L. Baldo, and M. E. Clark. 2008. Wolbachia: master manipulators of invertebrate biology. Nat. Rev. Microbiol. 6:741-751.

Williamson, D. L., R. F. Whitcomb, G. T. Joseph, G. E. Gasparich, D. L. Rose, P. Carle, J. M. Bové, K. J. Hackett, J. R. Adams, R. B. Henegar, et al. 1998. Revised group classification of the genus Spiroplasma. Int. J. Bacteriol. 48:1-12.

Woolfit, M., and L. Bromham. 2003. Increased rates of sequence evolution in endosymbiotic bacteria and fungi with small effective population sizes. Mol. Biol. Evol. 20:1545-1555.

Xie, J., S. Butler, G. Sanchez, and M. Mateos. 2014. Male killing Spiroplasma protects Drosophila melanogaster against two parasitoid wasps. Heredity 112:399-408.

Xie, J., I. Vilchez, and M. Mateos. 2010. Spiroplasma bacteria enhance survival of Drosophila hydei attacked by the parasitic wasp Leptopilina heterotoma. PLoS One 5:e12149.

Zchori-Fein, E., and S. J. Perlman. 2004. Distribution of the bacterial symbiont Cardinium in arthropods. Mol. Ecol. 13:2009-2016.

Zeng, F., G. Pederson, M. Ellsbury, and F. Davis. 1993. Demographic statistics for the pea aphid (Homoptera: Aphididae) on resistant and susceptible red clovers. J. Econ. Entomol. 86:1852-1856.

Associate Editor: K. King Handling Editor: M. Servedio

\section{Supporting Information}

Additional supporting information may be found at the end of the article.

Figure S1. Effect of the extraction protocol on the estimated number of aphid gene copies per aphid

Figure S2. Statistical power of phylogeny in Figure 5 for detecting phylogenetic signal.

Figure S3. Rate of emergence of the three wasp lines. 


\section{Supporting information}

Figure S1: Effect of the extraction protocol on the estimated number of aphid gene copies per aphid

The left and right panels show the absence of effect of the extraction kit on the estimated number of Spiroplasma genomes copy per aphid (y-axis), and the strong effect on the estimated number of aphid genomes copy per aphid (x-axis), estimations being much lower with the QIAGENE "DNeasy Blood \& Tissue Kit" (triangles) than with the QIAGENE "DNeasy 96 Blood \& Tissue Kit" (circles).
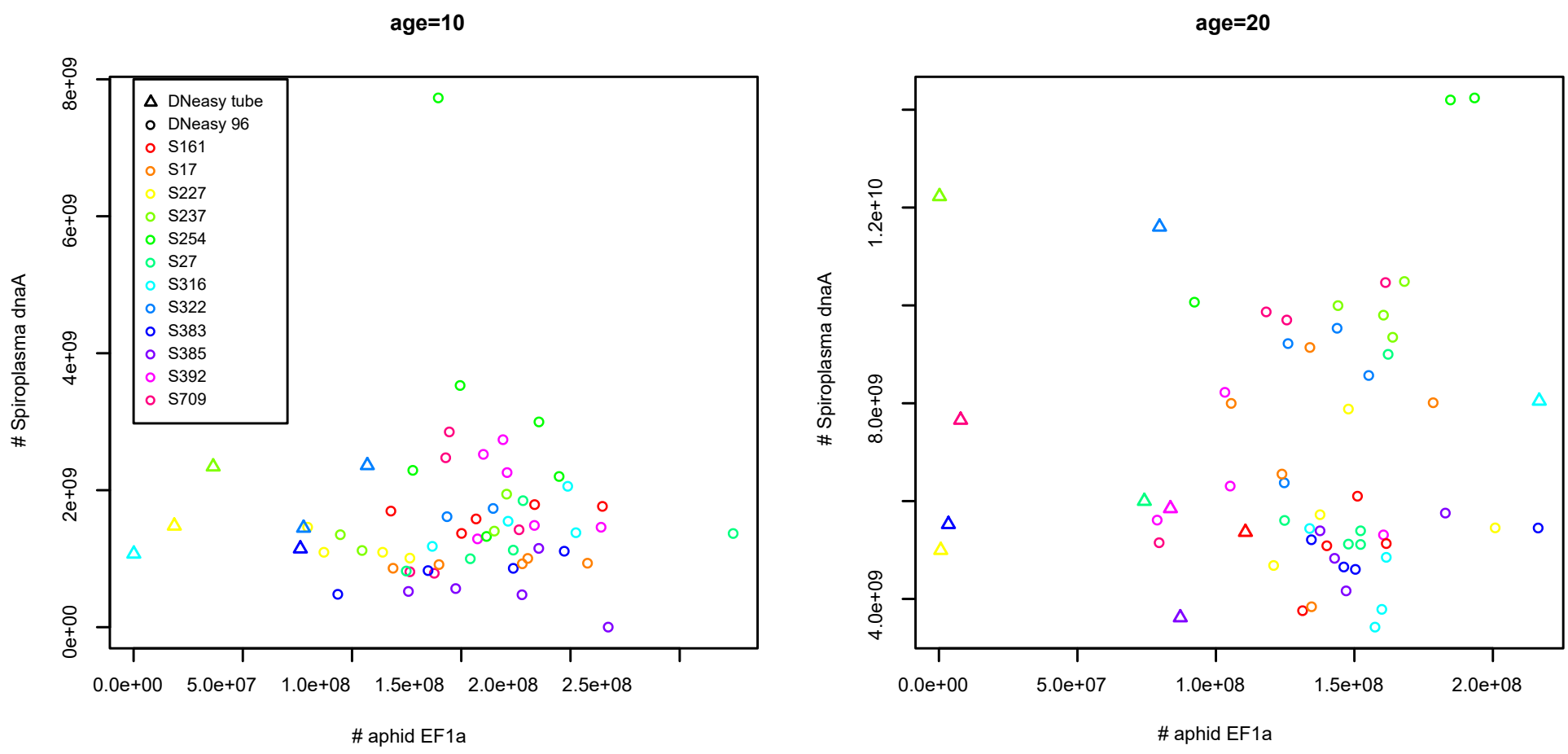
Figure S2: Statistical power of phylogeny in Figure 5 for detecting phylogenetic signal.

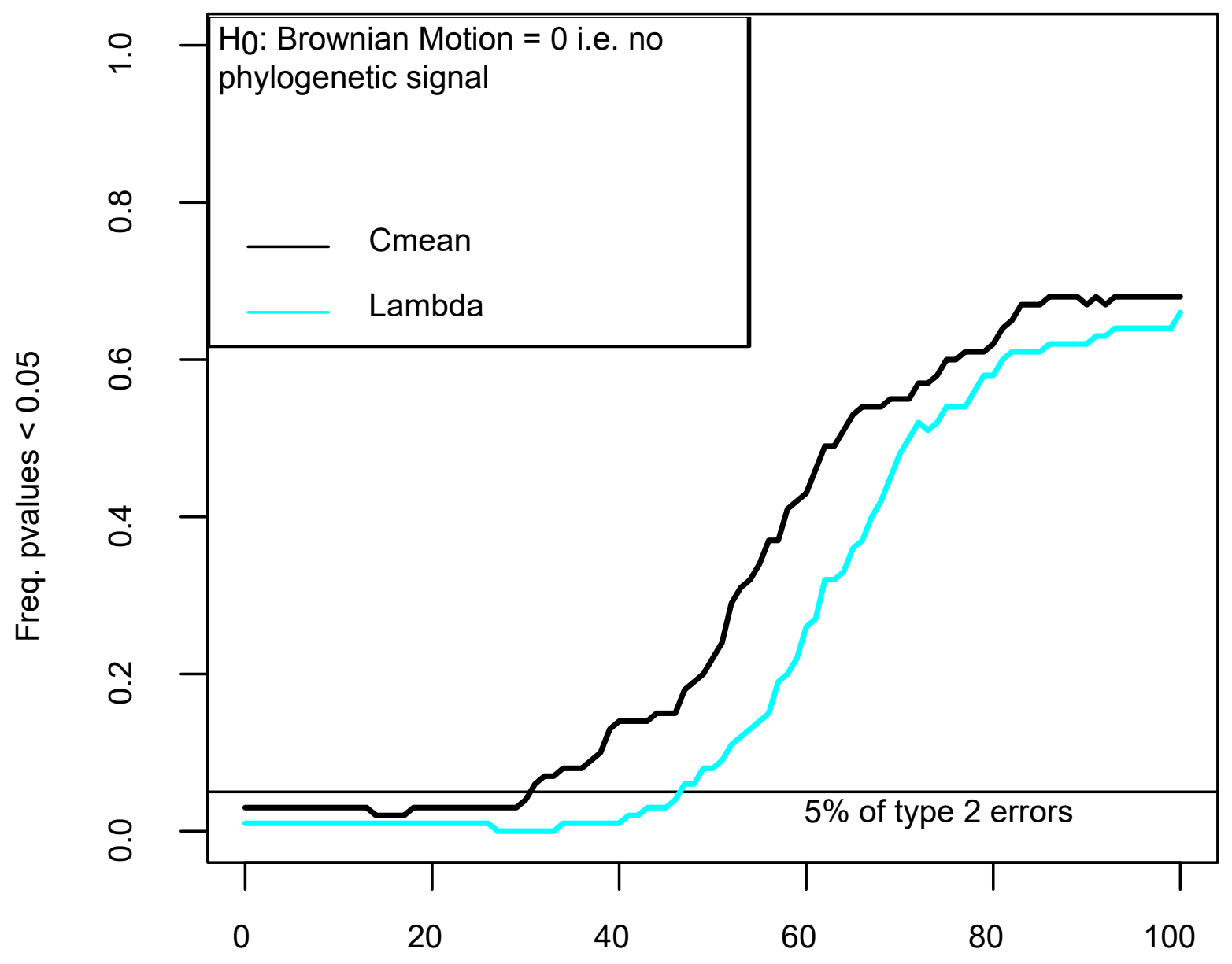

Brownian Motion (\%) 


\section{Figure S3: Rate of emergence of the three wasp lines.}

For each aphid sub-line, mean proportion of mummies from which the wasp succeeded their development, and emerged ( \pm S.E. indicated with error bars). Here, only the average over the three wasp lines is shown since the interaction wasp line $\times$ aphid sub-line is not significant. The bar S- corresponds to the control uninfected sub-line, and the bar S+ corresponds to the mean of all Spiroplasma infected sub-lines. Sub-lines also containing $R$. insecticola are hatched.

\section{Average over all lines}

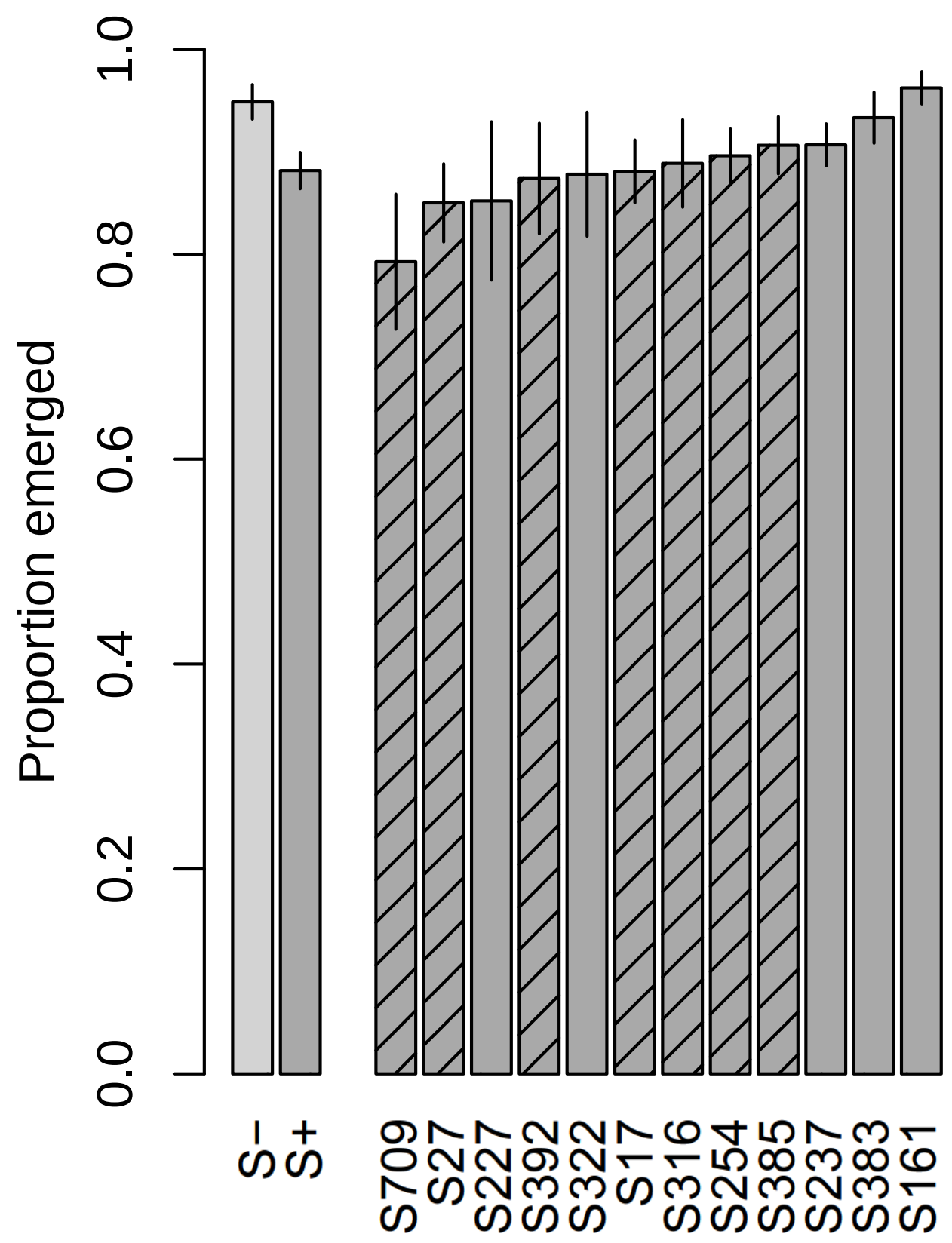

